ISSN 0103-9954

\title{
EXATIDÃO DOS MODELOS POLINOMIAIS NÃO-SEGMENTADOS E DAS RAZÕES ENTRE VOLUMES PARA REPRESENTAR O PERFIL DO TRONCO DE Pinus taeda
}

\section{ACCURACY OF NON-SEGMENTED POLYNOMIAL TAPER MODELS AND TAPER MODEL BASED ON VOLUME RATIO TO DESCRIBE THE STEM PROFILE OF Pinus taeda}

\author{
Fernando Fischer ${ }^{1}$ José Roberto S. Scolforo ${ }^{2}$ \\ Fausto Weimar Acerbi Júnior ${ }^{3}$ José Márcio de Mello ${ }^{3}$ Romualdo Maestri ${ }^{4}$
}

\begin{abstract}
RESUMO
O presente estudo teve como objetivos avaliar a: 1) exatidão das razões entre volumes compatíveis com sua respectiva função de afilamento; 2) os modelos polinomiais de potência inteira e dos modelos polinomiais de potências fracionárias e inteiras; 3) estudar a influência das classes de diâmetro, da produtividade dos sítios e das diferentes regiões na exatidão dos ajustes para representação do perfil do fuste de Pinus taeda na região nordeste do Paraná. Foram cubadas 304 árvores, utilizando-se do pentaprisma de Wheeler sobre tripé de base regulável com suunto acoplado. Nas proximidades de cada árvore cubada foi lançada uma parcela e medida a altura das árvores dominantes. Pôde-se efetuar assim a classificação de sítio no local de cada cubagem. Os diâmetros das árvores cubadas rigorosamente foram agrupados em classes, em intervalos de $5 \mathrm{~cm}$. Para avaliação da exatidão das estimativas do perfil do fuste, foram utilizadas as seguintes estatísticas: coeficiente de determinação; erro padrão residual; desvio dos diâmetros em cada i-ésima posição no fuste; desvio padrão das diferenças dos diâmetros; soma do quadrado do resíduo relativo e porcentagem dos resíduos dos diâmetros. Verificou-se que o ajuste das equações de afilamento devem ser preferidos por sítio e classe de diâmetro, além da utilização do modelo polinomial de potências fracionárias e inteiras para as estimativas do perfil do fuste.
\end{abstract}

Palavras-chave: funções de afilamento, perfil do tronco, Pinus taeda.

\footnotetext{
ABSTRACT

The presento study has had as its objective: 1) to evaluate the accuracy of the ratio between volumes compatible with its respective taper function; 2) to evaluate the entire polynomial models and entire and fractionary polynomial models; 3) to study the influence of the diameter classes, site productivity and local effects on the accuracy of the estimates provided by the taper models along

1. Engenheiro Florestal, MSc., Pesquisador do Instituto Estadual de Florestas de Minas Gerais, Av. Governador Valadares, 314, Bairro Centro, CEP 38610-000, Unaí (MG).

2. Engenheiro Florestal, Dr., Professor Titular do Departamento de Ciências Florestais, Universidade Federal de Lavras, Caixa Postal 37, CEP 37200-000, Lavras (MG).

3. Engenheiro Florestal, Dr., Professor Assitente do Departamento de Ciências Florestais, Universidade Federal de Lavras, Caixa Postal 37, CEP 37200-000, Lavras (MG).

4. Engenheiro Florestal, MSc., Pesquisador Sênior da Empresa Aracruz Celulose S.A., Rodovia Aracruz/Barra do Riacho, s/n, Caixa Postal 33.1011, CEP 29197-000, Aracruz (ES).
} 
the stem profiles of Pinus taeda in the north-eastern region of Parana State. It were cubed 304 trees using a Wheeler's calyper on tripold with a regulated base and with a suunto hypsometer. Near each cubed tree, it was located a plot from which the height of the dominant trees was measured, allowing the accomplishment of site classification of each cubage local. The diameters of cubed trees were assorted in classes with $5 \mathrm{~cm}$ intervals. For evaluating the accuracy of the taper models in describing the stem profile, the following statistics were calculated for each measurement position along the stem: coefficient of determination; residual standard error deviation; deviation of the diameters in each position along the stem; standard deviations of the differences of the diameters; sum of square of the relative residue of diameters and the percentage of the residues of the diameters. The main result pointed out that the taper models must be fitted by site productivity and diameter classes, besides the use of the polynomial model of fractionaire and entire power.

Key words: taper functions, stem profile, Pinus taeda.

\section{INTRODUÇÃO}

Muitos pesquisadores têm envidado esforços para obter um modelo que descreva com precisão o perfil do fuste de árvores, na expectativa de estimar o diâmetro a qualquer altura ao longo do fuste e a altura até qualquer diâmetro especificado. Esse dado permite quantificar o número de toras com bitola e comprimentos pré-definidos ou os múltiplos produtos da madeira. Normalmente, por meio da integração das funções de afilamento obtém-se expressões que permitem calcular o volume desses múltiplos produtos da madeira e mesmo de toda a árvore.

O primeiro trabalho para descrever o perfil do tronco foi feito por HOJER em 1903 e, após este, muitos outros modelos foram propostos. No Brasil, as funções de afilamento têm sido usadas desde 1970, e vários modelos foram utilizados para descrever o perfil do tronco, podendo-se citar trabalhos como o de SCHNEIDER (1986), LIMA (1986) e JORGE e LARA (1993), utilizando as funções de afilamento para quantificar o sortimento para Pinus elliottii; FRIEDL (1989) que comparou 3 modelos de afilamento para estudar a dinâmica da forma dos fustes de árvores de Araucaria angustifolia; ROSOT (1989) que comparou as equações de volumes tradicionais com as funções de forma por classe diamétrica e genéricas na estimativa do volume com casca por hectare; GUIMARÃES e LEITE (1992) que desenvolveram um novo modelo para descrever o perfil do tronco; SOARES (1993) que utilizou as funções de forma na otimização dos sortimentos de produtos florestais; CAMPOS et al. (1993) que utilizaram as funções de afilamento para comparar a forma de diferentes clones de Eucalyptus em duas regiões; FIGUEIREDO FILHO et al. (1993) que converteram equações de volume em equações de forma compatíveis para Pinus elliottii; GARCIA et al. (1993) que utilizaram as funções de forma para análise do perfil de árvores em diferentes espaçamentos; ANGELO et al. (1995) que utilizaram a função spline para definir a forma do tronco de Pinus tropicais; FINGER et al. (1995) que utilizaram as funções de forma para descrever o perfil do fuste de Eucalyptus dunnii; FIGUEIREDO FILHO et al. (1996) que compararam modelos segmentados e não-segmentados para descrever o perfil de Pinus taeda; RIOS (1997), FISCHER (1997), SCOLFORO et al. (1998) e FERREIRA (1999) que compararam modelos segmentados, não-segmentados, modelos de potência fracionária e de razões entre volumes e testaram a influência 
de fatores do meio nas estimativas volumétricas dos sortimentos e do perfil dos fustes de espécies florestais, dentre outros. De maneira geral, os trabalhos foram desenvolvidos buscando verificar, por meio de análise comparativa, qual modelo foi mais eficiente.

A aplicação dessas funções de afilamento é um poderoso instrumento para avaliar biológica e economicamente o maciço florestal e a resposta às práticas de manejo executadas, já que permite valorar de maneira detalhada quanto renderá o povoamento florestal.

O seu uso em escala comercial, no entanto, está condicionado à existência de softwares que tornem rápido o processamento do inventário florestal, com o objetivo de estimar os múltiplos produtos da madeira. Outro elemento de grande importância está em conhecer como a função de afilamento estima a base, o meio e o topo da árvore, principalmente para diferentes condições de ajuste.

Segundo FIGUEIREDO FILHO et al. (1996), o polinômio do $5^{\circ}$ grau tem sido o mais usado para descrever o perfil de Pinus taeda e Pinus elliotti na região sul do Brasil. O uso cada vez mais diversificado da madeira de Pinus sp nesta região para celulose, madeira serrada e madeira laminada, em diferentes bitolas e comprimentos, estimula cada vez mais o uso das funções de afilamento. No entanto, é necessário identificar quais variáveis podem auxiliar a melhorar a eficiência dessas estimativas. Estabeleceu-se então como objetivos deste trabalho, avaliar a eficiência das razões entre volumes compatíveis com a função de afilamento, o modelo polinomial de quinto grau e o de potências fracionárias e inteiras, e a influência das classes de diâmetro, da produtividade dos sítios e das diferentes regiões na exatidão dos ajustes para representar o perfil do fuste de Pinus taeda na região nordeste do Paraná.

\section{MATERIAL E MÉTODOS}

\section{Base de dados}

A área em estudo, de propriedade da Empresa Pisa Papel Imprensa S.A., está situada no município de Jaguariaíva, estado do Paraná, entre os paralelos $24^{\circ}$ e $24^{\circ} 30^{\prime}$ de latitude sul e os meridianos $49^{\circ} 30^{\prime}$ e $50^{\circ}$ de longitude oeste de Greenwich, com altitude variando entre 700 e 1.100 metros.

Foram cubadas rigorosamente 304 árvores de Pinus taeda distribuídas em cinco regiões da empresa, nas quais foram medidos os diâmetros nas alturas relativas de 1, 2, 3, 4, 5, 10, 15, 25, 35, $\ldots, 95 \%$ da altura total da árvore bem como o diâmetro a $1,3 \mathrm{~m}$ do solo (DAP) e a altura total da árvore (H). Para as alturas relativas de 1, 2, 3, 4, 5\%, e a 1,30 m do solo, os diâmetros foram medidos diretamente com fita métrica. Para as demais alturas, utilizou-se o penta-prisma de Wheeler sobre tripé de base regulável e com suunto acoplado, o que possibilitou a medição desses diâmetros com as árvores em pé.

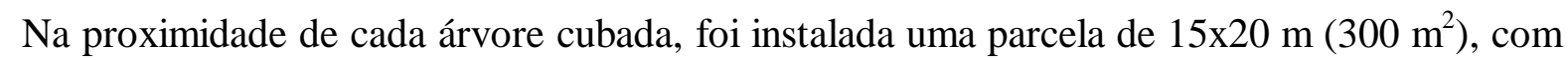
objetivo de identificar a altura dominante. Foi mensurada a altura das três árvores mais grossas da 
parcela, resultando na altura média das árvores dominantes. De posse de cada par de altura dominante-idade foi possível identificar o índice de sítio do local onde foi cubada cada árvore. A equação de sítio utilizada foi desenvolvida por SCOLFORO (1997) e apresentou coeficiente de determinação $\left(R^{2}\right)$ igual a 97,48\% e erro padrão residual (Syx) igual a 1,187 m. A equação é:

$$
\mathrm{Hd}=52,3735174 .\left(\frac{\mathrm{S}}{52,3735174}\right)^{\frac{\ln (1-\exp (-0,0275482 . \mathrm{I}))}{\ln (1-\exp (-0,0275482 . \text { Iref }))}}
$$

A Tabela 1 apresenta a distribuição das árvores cubadas por classe diamétrica e índice de sítio.

TABELA 1: Distribuição das árvores por classe diamétrica nos diferentes sítios.

\begin{tabular}{c|c|c|c|c|c}
\hline Classe diamétrica & I & II & III & IV & V \\
\hline $10-15$ & 0 & 3 & 1 & 0 & 0 \\
$15-20$ & 3 & 12 & 8 & 5 & 2 \\
$20-25$ & 2 & 23 & 18 & 12 & 16 \\
$25-30$ & 1 & 14 & 16 & 12 & 15 \\
$30-35$ & 1 & 8 & 16 & 12 & 14 \\
$35-40$ & 1 & 11 & 17 & 12 & 8 \\
$40-45$ & 2 & 4 & 16 & 7 & 2 \\
$45-50$ & 0 & 1 & 3 & 1 & 1 \\
$50-55$ & 0 & 1 & 2 & 0 & 0 \\
$55-60$ & 0 & 1 & 0 & 0 & 0 \\
\hline TOTAL & 10 & 78 & 97 & 61 & 58 \\
\hline
\end{tabular}

\section{Distribuição diamétrica}

$\mathrm{Na}$ Tabela 2, estão apresentadas por classe diamétrica e por região de estudo, a freqüência das árvores cubadas rigorosamente.

TABELA 2: Distribuição das árvores por classe diamétrica e região.

\begin{tabular}{c|c|c|c|c|c}
\hline Classe diamétrica & Região 1 & Região 2 & Região 3 & Região 4 & Região 5 \\
\hline $10-15$ & 0 & 0 & 2 & 0 & 2 \\
$15-20$ & 3 & 5 & 8 & 2 & 12 \\
$20-25$ & 15 & 13 & 13 & 12 & 18 \\
$25-30$ & 13 & 14 & 15 & 11 & 5 \\
$30-35$ & 12 & 10 & 12 & 12 & 5 \\
$35-40$ & 13 & 9 & 8 & 13 & 6 \\
$40-45$ & 6 & 7 & 4 & 9 & 5 \\
$45-50$ & 0 & 2 & 2 & 1 & 1 \\
$50-55$ & 0 & 2 & 1 & 0 & 0 \\
$55-60$ & 0 & 1 & 0 & 0 & 0 \\
\hline TOTAL & 62 & 63 & 65 & 60 & 54 \\
\hline
\end{tabular}




\section{Funções de afilamento}

Utilizou-se o polinômio do $5^{\circ}$ grau desenvolvido por SCHOPFER (1966), o polinômio de potências fracionárias e inteiras apresentado por HRADETZKY (1976) e também as razões entre volumes compatíveis, conforme apresentado por CLUTTER (1980), e AMATEIS e BURKHART (1987).

Nos modelos polinomiais, a variável dependente é expressa pela razão entre o diâmetro tomado ao longo do fuste $\left(\mathrm{d}_{\mathrm{i}}\right)$ e o diâmetro a $1,30 \mathrm{~m}$ (D) ou diâmetro a $10 \%$ da altura $\left(\mathrm{D}_{0,1}\right)$, ou seja $\left(\frac{d_{i}}{D}\right)$. Já as variáveis independentes caracterizam-se pela razão entre a altura tomada ao longo do fuste $\left(h_{i}\right)$ e a altura total $(H)$ elevada a potência pi, ou seja $\left(\frac{h_{i}^{p i}}{H^{p i}}\right)$.

No caso do polinômio do $5^{\circ}$ grau, essas potências são $1 ; 2 ; 3 ; 4$ e 5.

O modelo de potências fracionárias e inteiras deve ser construído para cada situação de trabalho. Neste estudo, foi utilizado o método "Stepwise" em que foram consideradas, no modelo cheio, as seguintes potências: 0,$005 ; 0,09 ; 0,08 ; 0,07 ; 0,06 ; 0,05 ; 0,04 ; 0,03 ; 0,02 ; 0,01 ; 0,9 ; 0,8$; 0,$7 ; 0,6 ; 0,5 ; 0,4 ; 0,3 ; 0,2 ; 0,1 ; 1 ; 2 ; 3 ; 4 ; 5 ; 10 ; 15 ; 20$ e 25 . A expectativa é que as potências (pi) inteiras unitárias representem melhor a seção intermediária da árvore, as potências com grandezas de dezenas a base da árvore, e as potências fracionárias o topo da árvore.

No caso do modelo de CLUTTER (1980), o fundamento é que uma equação de volume comercial de topo variável define a função de afilamento, considerando o modelo de Schumacher e Hall $\left(V=\beta_{0} D^{\beta 1} H^{\beta 2}+e_{i}\right)$ e a razão entre volumes $\left[V_{C}=V\left(1-\alpha_{0} d^{\alpha_{1}} D^{\alpha_{2}}\right)\right]$. Aplicando o conceito de integral e derivada, obtém-se a função de afilamento que propicia obter a altura correspondente a qualquer diâmetro e o diâmetro a qualquer altura, como mostrado na Tabela 3.

No caso do modelo de AMATEIS e BURKHART (1987), são utilizadas duas razões entre volumes, sendo que uma das razões $\left[\mathrm{v}_{\mathrm{c}}=\mathrm{V}\left(1-\alpha_{0} \frac{\mathrm{d}^{\alpha_{1}}}{\mathrm{D}^{\alpha_{2}}}\right)\right]$ é função do diâmetro e a outra $\left[\mathrm{v}_{\mathrm{c}}=\mathrm{V}\left(1-\beta_{0} \frac{(\mathrm{H}-\mathrm{h})^{\beta_{1}}}{\mathrm{H}^{\beta_{2}}}\right)\right]$ é função da altura. Igualando as funções $\alpha_{0} \frac{\mathrm{d}^{\alpha_{1}}}{\mathrm{D}^{\alpha_{2}}}=\beta_{0} \frac{(\mathrm{H}-\mathrm{h})^{\beta_{1}}}{\mathrm{H}^{\beta_{2}}}$ e isolando o $d_{i}$, tem-se a função de afilamento que propicia obter o diâmetro a qualquer altura. Se for isolada a variável altura, obtém-se a função que propicia obter a altura correspondente a qualquer diâmetro.

$\mathrm{Na}$ Tabela 3, estão apresentadas as formas de ajustes e as funções de afilamento que propiciam implementar o estudo em questão. O ajuste das funções de afilamento polinomiais foi efetuado por classe diamétrica para cada região considerada neste estudo e também para cada classe de sítio. Já o ajuste das razões entre volumes e a equação de volume total foram efetuadas para região e também por classe de sítio. 
TABELA 3: Formas de ajuste e de uso dos polinômios e razões de volume.

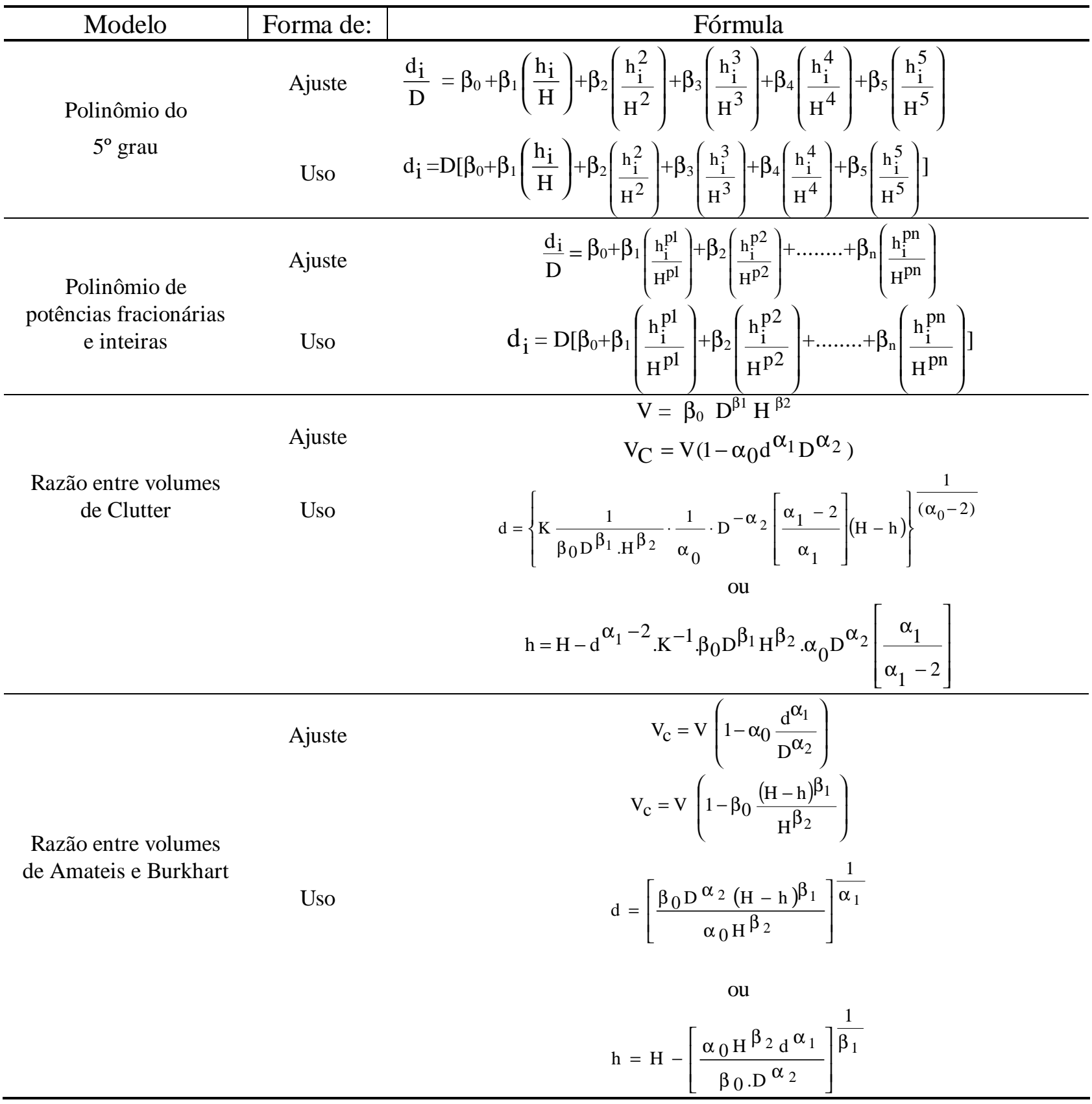

Sendo: $\mathrm{V}=$ volume total $\mathrm{da}$ árvore; $\mathrm{V}_{\mathrm{c}}=$ volume correspondente a qualquer diâmetro mínimo pré-estabelecido ao longo do fuste; $\mathrm{H}=$ altura total da árvore; $\mathrm{h}_{\mathrm{i}}=$ altura correspondente a qualquer diâmetro $\left(\mathrm{d}_{\mathrm{i}}\right)$ ao longo do fuste, no caso dos modelos polinomiais e ao diâmetro mínimo ao longo do fuste previamente estabelecido, no caso das razões de volume; $d_{i}=$ diâmetro correspondente a qualquer altura $\left(h_{i}\right)$ ao longo do fuste, no caso dos modelos polinomiais e às alturas vinculadas aos diâmetros mínimos comerciais previamente estabelecidos, no caso das razões de volume; $\alpha_{i}$ e $\beta_{i}$ = parâmetros a serem estimados. 
Exatidão dos modelos de afilamento para representar o perfil do tronco, por classe de diâmetro, região e sítio

Para avaliar a exatidão dessas estimativas, foram adotados os critérios utilizados em PARRESOL et al. (1987), FIGUEIREDO FILHO et al. (1996) e SCOLFORO et al. (1998), conforme apresentado na Tabela 4. Foi utilizado um número semelhante de repetições que será de cinco árvores por classe de diâmetro, em cada região e sítio.

Em consequiência do pequeno número de repetições nas classes diamétricas de 10 a 15, 15 a 20, 40 a 45, 45 a 50, 50 a 55, 55 a 60, estas foram eliminadas neste estudo.

TABELA 4: Estatísticas utilizadas para avaliação das estimativas dos diâmetros.

\begin{tabular}{ll}
\hline Estimativa & Fórmula \\
\hline Desvio (D) & $\left(\sum\right.$ Diff $\left._{\mathrm{i}}\right) / \mathrm{N}$ \\
Desvio padrão das diferenças (SD) & {$\left[\sum\left(\mathrm{Diff}_{\mathrm{i}}-\mathrm{D}\right)^{2} /(\mathrm{N}-1)\right]^{0.5}$} \\
Soma do quadrado do resíduo relativo (SSRR) & $\sum\left(\mathrm{Diff}_{\mathrm{i}} / \mathrm{d}_{\mathrm{i}}\right)^{2}$ \\
Porcentagem dos resíduos (RP) & $\sum\left(\text { Diff }_{\mathrm{i}} / \mathrm{d}_{\mathrm{i}}\right)^{*} 100 / \mathrm{N}$ \\
\hline
\end{tabular}

Em que: Diff ${ }_{\mathrm{i}}=\left(\mathrm{d}_{\mathrm{i}}-\mathrm{de}_{\mathrm{i}}\right)$ é o desvio dos diâmetros em cada i-ésima posição no fuste $(1 \%, 2 \%, \ldots, 95 \%) ; \mathrm{d}_{\mathrm{i}}=$ diâmetro da árvore, na posição i no fuste; $\mathrm{de}_{\mathrm{i}}=$ diâmetro da árvore estimado para a posição i no fuste; $\mathrm{N}=$ número de árvores.

Com base nestas estatísticas, foi elaborado um ranking para expressar de forma sumarizada a exatidão dos quatro modelos de afilamento testados. Esse ranking foi feito para cada posição relativa em que se efetuou a cubagem rigorosa e também por classe diamétrica, considerando as quatro estatísticas da Tabela 4. Foi atribuída a nota 1 à equação que apresentou maior exatidão e assim sucessivamente, até a nota 4 para a equação que apresentou menor exatidão. No caso de mesmo valor em qualquer das estatísticas, para diferentes equações estas receberão a mesma nota.

\section{RESULTADOS E DISCUSSÃO}

\section{Ajuste do polinômio de potências fracionárias e inteiras por classe diamétrica, região e sítio}

As Tabelas 5 e 6 apresentam o ajuste para esses polinômios, respectivamente, para diferentes classes de sítio e regiões. Pode-se observar que os primeiros parâmetros são potências fracionárias e os parâmetros finais dos modelos são potências inteiras, geralmente com grandeza de dezena. Os modelos com quatro coeficientes apresentaram, na maioria dos casos, a potência 0,005 no segundo parâmetro, potências decimais para o terceiro parâmetro, unitárias para o quarto e de dezenas no último parâmetro. Nesses casos, as potências fracionárias estão moldando melhor a ponta da árvore e as potências maiores a base da árvore. Pôde-se constatar que, para todas as situações testadas, as medidas de precisão estão dentro do espectro de ajustes precisos.

Por meio das medidas de precisão não foram detectadas diferenças entre as equações ajustadas para as diversas classes diamétricas. Quanto aos ajustes realizados por sítio e classe diamétrica, e região e classe diamétrica, verificou-se que os menores e maiores valores de coeficiente de determinação foram respectivamente 94,9 e 99,4\%; e 93,6 e 98,8\%. Juntamente com o erro padrão residual, pôde-se inferir que há uma ligeira superioridade dos modelos ajustados por sítio em relação às equações obtidas por região em consequiência provavelmente, do maior controle das 
fontes de variação da variável volume.

TABELA 5: Parâmetros estimados e medidas de precisão do polinômio de potência fracionária e inteira por classe de diâmetro e por sítio.

\begin{tabular}{|c|c|c|c|c|c|c|c|c|c|c|c|}
\hline \multirow[t]{2}{*}{$\mathrm{S}$} & \multirow{2}{*}{$\begin{array}{l}\text { CLD } \\
(\mathrm{cm})\end{array}$} & \multirow{2}{*}{$\begin{array}{l}\mathrm{P} / \\
\mathrm{C}\end{array}$} & \multicolumn{9}{|c|}{ Parâmetros estimados } \\
\hline & & & $\beta_{0}$ & $\beta_{1}$ & $\beta_{2}$ & $\beta_{3}$ & $\beta_{4}$ & $\beta_{5}$ & $\beta_{6}$ & $\mathrm{R}^{2}$ & Syx \\
\hline \multirow{12}{*}{ II } & $15+20$ & $\mathrm{P}$ & & 0.005 & 0.01 & 0.5 & 0.7 & 3 & 10 & & \\
\hline & & $\mathrm{C}$ & 1,35865 & 28,09798 & $-28,92649$ & 1,49566 & $-1,68955$ & $-0,32113$ & 0,91601 & 97,5 & 0,0467 \\
\hline & $20+25$ & $\mathrm{P}$ & & 0,005 & 0,5 & 0,6 & 0,7 & 4 & 10 & & \\
\hline & & $\mathrm{C}$ & 1,35173 & 0,30110 & $-19,90846$ & 37,13755 & $-18,54327$ & $-0,6621$ & 1,22302 & 97,3 & 0,0508 \\
\hline & $25+30$ & $\mathrm{P}$ & & 0,005 & 0,01 & 0,6 & 4 & 10 & & & \\
\hline & & $\mathrm{C}$ & 1,29232 & 13,66334 & $-14,05647$ & $-0,44580$ & $-0,87933$ & 1,22495 & & 96,8 & 0,0548 \\
\hline & $30+35$ & $\mathrm{P}$ & & 0,005 & 0,5 & 10 & 15 & & & & \\
\hline & & $\mathrm{C}$ & 1,34488 & $-0,13397$ & $-0,84765$ & $-2,7877$ & 4,48199 & & & 97,0 & 0,0529 \\
\hline & $35 \vdash 40$ & $\mathrm{P}$ & & 0,005 & 0,3 & 5 & 10 & & & & \\
\hline & & $\mathrm{C}$ & 1,26673 & 0,08493 & $-0,83895$ & $-1,12667$ & 1,02467 & & & 98,1 & 0,0408 \\
\hline & $40+45$ & $\mathrm{P}$ & & 0,005 & 0,5 & 0,6 & 2 & & & & \\
\hline & & $\mathrm{C}$ & 1,33280 & 0,25242 & $-9,44728$ & 9,28210 & $-1,46446$ & & & 98,3 & 0,0405 \\
\hline \multirow{12}{*}{ III } & 15 卜 20 & $\mathrm{P}$ & & 0,5 & 3 & & & & & & \\
\hline & & $\mathrm{C}$ & 1,30814 & $-0,92740$ & $-0,17720$ & & & & & 95,4 & 0,0612 \\
\hline & $20+25$ & $\mathrm{P}$ & & 0,005 & 0,06 & 0,6 & 3 & 10 & & & \\
\hline & & $\mathrm{C}$ & 1,28912 & 1,16649 & $-1,57761$ & $-0,37797$ & $-0,64520$ & 0,93303 & & 95,6 & 0,0615 \\
\hline & $25+30$ & $\mathrm{P}$ & & 0,005 & 0,01 & 1 & 5 & & & & \\
\hline & & $\mathrm{C}$ & 1,27835 & 14,73885 & $-15,19333$ & $-0,50575$ & $-0,26530$ & & & 97,7 & 0,0446 \\
\hline & $30+35$ & $\mathrm{P}$ & & 0,005 & 0,3 & 5 & 10 & & & & \\
\hline & & $\mathrm{C}$ & 1,31752 & 0,06122 & $-0,85365$ & $-1,02847$ & 0,82673 & & & 97,4 & 0,048 \\
\hline & $35 \vdash 40$ & $\mathrm{P}$ & & 0,005 & 0,2 & 4 & 10 & 20 & 25 & & \\
\hline & & $\mathrm{C}$ & 1,28798 & 0,28202 & $-0,99707$ & $-0,90670$ & 1,07736 & $-4,46712$ & 4,74722 & 97,9 & 0,0433 \\
\hline & $40+45$ & $\mathrm{P}$ & & 0,005 & 0,2 & 0,3 & 0,7 & 4 & 10 & & \\
\hline & & $\mathrm{C}$ & 1,26463 & 1,38212 & $-8,59846$ & 8,13193 & $-1,70846$ & $-0,83277$ & 0,61998 & 97,7 & 0,0458 \\
\hline \multirow{12}{*}{ IV } & $15 \vdash 20$ & $\mathrm{P}$ & & 0,4 & 4 & & & & & & \\
\hline & & C & 1,39705 & $-0,99823$ & $-0,20731$ & & & & & 96,5 & 0,0579 \\
\hline & $20+25$ & $\mathrm{P}$ & & 0,4 & 5 & 10 & & & & & \\
\hline & & $\mathrm{C}$ & 1,32274 & $-0,86223$ & $-0,86293$ & 1,06299 & & & & 97,2 & 0,0485 \\
\hline & $25+30$ & $\mathrm{P}$ & & 0,005 & 0,3 & 0,4 & 0,7 & 5 & & & \\
\hline & & $\mathrm{C}$ & 1,33175 & 1,10031 & $-15,59416$ & 18,52482 & $-5,10074$ & $-0,22113$ & & 98,3 & 0,0388 \\
\hline & $30+35$ & $\mathrm{P}$ & & 0,005 & 0,2 & 0,6 & 1 & 5 & & & \\
\hline & & $\mathrm{C}$ & 1,31812 & 0,85796 & $-2,84394$ & 3,26568 & $-2,35021$ & $-0,26552$ & & 97,1 & 0,0512 \\
\hline & $35 \vdash 40$ & $\mathrm{P}$ & & 0,005 & 0,2 & 4 & 5 & & & & \\
\hline & & $\mathrm{C}$ & 1,26321 & 0,24047 & $-0,85064$ & $-1,79405$ & 1,13322 & & & 98,1 & 0,0395 \\
\hline & $40+45$ & $\mathrm{P}$ & & 0,5 & 0,6 & 5 & 10 & & & & \\
\hline & & $\mathrm{C}$ & 1,35209 & $-3,04101$ & 2,28350 & $-1,59701$ & 1,64839 & & & 94,9 & 0,0743 \\
\hline \multirow{12}{*}{ V } & $15 \vdash 20$ & $\mathrm{P}$ & & 0,5 & & & & & & & \\
\hline & & $\mathrm{C}$ & 1,36622 & $-1,036$ & & & & & & 98,6 & 0,0358 \\
\hline & $20+25$ & $\mathrm{P}$ & & 0,4 & 5 & 10 & & & & & \\
\hline & & $\mathrm{C}$ & 1,32419 & $-0,84830$ & $-0,95944$ & 1,09523 & & & & 96,5 & 0,0543 \\
\hline & $25+30$ & $\mathrm{P}$ & & 0,4 & 1 & 5 & 25 & & & & \\
\hline & & $\mathrm{C}$ & 1,32888 & $-0,92493$ & 0,11542 & $-0,85590$ & 5,95237 & & & 98,1 & 0,0413 \\
\hline & $30+35$ & $\mathrm{P}$ & & 0,005 & 0,3 & 5 & 10 & & & & \\
\hline & & $\mathrm{C}$ & 1,30035 & 0,10635 & $-0,87794$ & $-1,22562$ & 1,15233 & & & 97,6 & 0,0477 \\
\hline & $35 \vdash 40$ & $\mathrm{P}$ & & 0,4 & 5 & & & & & & \\
\hline & & $\mathrm{C}$ & 1,29850 & $-0,82603$ & $-0,64228$ & & & & & 98 & 0,0426 \\
\hline & $40+45$ & $\mathrm{P}$ & & 0,005 & 0,02 & 1 & 5 & 25 & & & \\
\hline & & $\mathrm{C}$ & 1,25621 & 4,82308 & $-5,28019$ & $-0,20437$ & $-1,27062$ & 8,84420 & & 99,4 & 0,0234 \\
\hline
\end{tabular}

Em que: $\mathrm{S}=$ Classes de sítio; $\mathrm{CLD}=$ Classes de diâmetro; $\mathrm{P}=$ Potência; $\mathrm{C}=$ Coeficiente. 
TABELA 6: Parâmetros estimados e medidas de precisão do polinômio de potência fracionária e inteira por classe de diâmetro e por região.

\begin{tabular}{|c|c|c|c|c|c|c|c|c|c|c|c|}
\hline \multirow[t]{2}{*}{$\mathrm{R}$} & \multirow{2}{*}{$\begin{array}{l}\text { CLD } \\
(\mathrm{cm})\end{array}$} & \multirow{2}{*}{$\begin{array}{l}\mathrm{P} / \\
\mathrm{C}\end{array}$} & \multicolumn{9}{|c|}{ Parâmetros estimados } \\
\hline & & & $\beta_{0}$ & $\beta_{1}$ & $\beta_{2}$ & $\beta_{3}$ & $\beta_{4}$ & $\beta_{5}$ & $\beta_{6}$ & $\mathrm{R}^{2}$ & Syx \\
\hline \multirow{13}{*}{1} & $15-20$ & $\mathrm{P}$ & & 0,4 & 5 & & & & & & \\
\hline & & $\mathrm{C}$ & 1,36974 & $-0,99538$ & $-0,13684$ & & & & & 98,1 & 0,042 \\
\hline & $20+25$ & $\mathrm{P}$ & & 0,005 & 0,01 & 0,6 & 0,5 & 5 & 10 & & \\
\hline & & $\mathrm{C}$ & 1,29440 & 28,22982 & $-29,09063$ & $-3,08218$ & 3,01034 & $-0,45615$ & 0,54750 & 97,0 & 0,049 \\
\hline & $25-30$ & $\mathrm{P}$ & & 0,005 & 0,01 & 0,5 & 0,6 & 4 & 5 & & \\
\hline & & $\mathrm{C}$ & 1,26663 & 40,74125 & $-42,02229$ & 5,82576 & $-5,46071$ & 0,10649 & - & 97,8 & 0,041 \\
\hline & & & & & & & & & 0,39353 & & \\
\hline & $30+35$ & $\mathrm{P}$ & & 0,005 & $\begin{array}{c}0,01 \\
76021\end{array}$ & 0,2 & 0,7 & 5 & & & \\
\hline & & $\mathrm{C}$ & 1,26128 & 72,77773 & $-76,09311$ & 3,76866 & $-1,32512$ & $-0,41769$ & & 98,2 & 0,034 \\
\hline & $35-40$ & $\mathrm{P}$ & & 0,005 & 0,3 & 5 & & & & & \\
\hline & & $\mathrm{C}$ & 1,27283 & 0,09811 & $-0,82695$ & $-0,73295$ & & & & 97,6 & 0,044 \\
\hline & $40+45$ & $\mathrm{P}$ & & 0,005 & 0,4 & 0,5 & 0,7 & 5 & 10 & & \\
\hline & & $\mathrm{C}$ & 1,25350 & 0,55537 & $-14,61717$ & 19,61877 & $-6,31274$ & $-1,41646$ & 1,56867 & 98,4 & 0,037 \\
\hline \multirow{12}{*}{2} & 15 - 20 & $\mathrm{P}$ & & 0,5 & & & & & & & \\
\hline & & $\mathrm{C}$ & 1,32189 & $-1,03361$ & & & & & & 96,6 & 0,0532 \\
\hline & $20+25$ & $\mathrm{P}$ & & 0,005 & 0,01 & 0,6 & 3 & 5 & & & \\
\hline & & $\mathrm{C}$ & 1,28193 & 14,83978 & $-15,26079$ & $-0,28629$ & $-1,42619$ & 1,28959 & & 97,8 & 0,043 \\
\hline & $25+30$ & $\mathrm{P}$ & & 0,005 & 0,2 & 0,5 & 1 & 4 & 15 & & \\
\hline & & $\mathrm{C}$ & 1,28542 & 0,52570 & $-1,88337$ & 1,36408 & $-1,04408$ & $-0,25397$ & 0,86905 & 97,7 & 0,0453 \\
\hline & $30+35$ & $\mathrm{P}$ & & 0,2 & 0,5 & 5 & 20 & & & & \\
\hline & & $\mathrm{C}$ & 1,31992 & $-0,34002$ & $-0,54102$ & $-0,56901$ & 0,69242 & & & 97,5 & 0,0471 \\
\hline & $35+40$ & $\mathrm{P}$ & & 0,3 & 0,6 & 5 & 15 & & & & \\
\hline & & $\mathrm{C}$ & 1,26959 & $-0,46916$ & $-0,37506$ & $-0,67713$ & 0,65161 & & & 97,9 & 0,0434 \\
\hline & $40+45$ & $\mathrm{P}$ & & 0,005 & 0,01 & 4 & 5 & & & & \\
\hline & & $\mathrm{C}$ & 1,23918 & 20,94162 & $-21,47465$ & $-3,11928$ & 2,61393 & & & 93,6 & 0,08 \\
\hline \multirow{12}{*}{3} & 15 - 20 & $\mathrm{P}$ & & 0,005 & 0,2 & 0,6 & 4 & 5 & & & \\
\hline & & $\mathrm{C}$ & 1,32362 & 0,51321 & $-1,44239$ & 0,30586 & $-5,15998$ & 5,48307 & & 96,6 & 0,0541 \\
\hline & $20+25$ & $\mathrm{P}$ & & 0,5 & 1 & 3 & 15 & & & & \\
\hline & & $\mathrm{C}$ & 1,31547 & $-1,59976$ & 1,19345 & $-1,61956$ & 14,95960 & & & 96,4 & 0,0583 \\
\hline & $25+30$ & $\mathrm{P}$ & & 0,005 & 0,01 & 0,3 & 0,6 & 5 & 25 & & \\
\hline & & $\mathrm{C}$ & 1,29942 & 45,13724 & $-46,84223$ & 2,52066 & $-1,72575$ & $-0,73471$ & 7,03981 & 97,7 & 0,0461 \\
\hline & $30+35$ & $\mathrm{P}$ & & 0,005 & 0,01 & 0,3 & 2 & 5 & 15 & & \\
\hline & & $\mathrm{C}$ & 1,30545 & 28,02768 & $-28,86725$ & 0,34990 & $-0,64813$ & $-0,36173$ & 0,79620 & 98,2 & 0,0412 \\
\hline & 35 - 40 & $\mathrm{P}$ & & 0,005 & 0,01 & 4 & 5 & 10 & 25 & & \\
\hline & & $\mathrm{C}$ & 1,26930 & 19,98036 & $-20,54010$ & $-4,51801$ & 4,98054 & $-1,81337$ & 1,66415 & 98,8 & 0,0325 \\
\hline & $40+45$ & $\mathrm{P}$ & & 0,005 & 0,2 & 5 & 10 & & & & \\
\hline & & $\mathrm{C}$ & 1,32075 & 0,19351 & $-0,90048$ & $-1,74490$ & 1,76620 & & & 98,7 & 0,0357 \\
\hline \multirow{3}{*}{4} & 15 卜 20 & $\mathrm{P}$ & & 0,4 & 10 & & & & & & \\
\hline & & $\mathrm{C}$ & 1,45979 & $-1,06857$ & $-2,16114$ & & & & & 97,5 & 0,0522 \\
\hline & $20-25$ & $\mathrm{P}$ & & 0,4 & 5 & 10 & & & & & \\
\hline
\end{tabular}

Continua ... 
TABELA 6: Continuação ...

\begin{tabular}{|c|c|c|c|c|c|c|c|c|c|c|c|}
\hline \multirow[t]{2}{*}{$\mathrm{R}$} & \multirow{2}{*}{$\begin{array}{l}\text { CLD } \\
(\mathrm{cm})\end{array}$} & \multirow{2}{*}{$\begin{array}{l}\mathrm{P} / \\
\mathrm{C}\end{array}$} & \multicolumn{9}{|c|}{ Parâmetros estimados } \\
\hline & & & $\beta_{0}$ & $\beta_{1}$ & $\beta_{2}$ & $\beta_{3}$ & $\beta_{4}$ & $\beta_{5}$ & $\beta_{6}$ & \multirow{2}{*}{$\frac{\mathrm{R}^{2}}{97,6}$} & \multirow{2}{*}{$\frac{\text { Syx }}{0,0447}$} \\
\hline \multirow{9}{*}{4} & \multirow{3}{*}{$25+30$} & $\mathrm{C}$ & 1,32513 & $-0,87736$ & $-0,84185$ & 0,88859 & & & & & \\
\hline & & $\mathrm{P}$ & & 0,005 & 0,3 & 5 & 10 & & & & \\
\hline & & $\mathrm{C}$ & 1,33638 & 0,09490 & $-0,93398$ & $-1,16469$ & 1,23253 & & & 98,3 & 0,04 \\
\hline & $30+35$ & $\mathrm{P}$ & & 0,005 & 0,01 & 3 & 5 & & & & \\
\hline & & $\mathrm{C}$ & 1,33281 & 22,37937 & $-23,03295$ & $-1,20054$ & 0,62540 & & & 98,3 & 0,0416 \\
\hline & $35+40$ & $\mathrm{P}$ & & 0,005 & 0,4 & 0,8 & 1 & 5 & 10 & & \\
\hline & & $\mathrm{C}$ & 1,28417 & 0,20168 & $-2,52294$ & 5,31669 & $-3,88987$ & $-0,85972$ & 0,73677 & 98,5 & 0,0368 \\
\hline & $40+45$ & $\mathrm{P}$ & & 0,005 & 0,01 & 0,3 & 5 & 15 & & & \\
\hline & & $\mathrm{C}$ & 1,31140 & 11,62333 & $-11,93089$ & $-0,42131$ & $-1,40515$ & 2,49657 & & 98,2 & 0,0423 \\
\hline \multirow{12}{*}{5} & 15 - 20 & $\mathrm{P}$ & & 0,005 & 0,1 & 0,5 & 3 & 5 & & & \\
\hline & & $\mathrm{C}$ & 1,36704 & 0,95489 & $-1,62319$ & $-0,06627$ & $-1,19393$ & 1,06462 & & 97,3 & 0,0477 \\
\hline & $20+25$ & $\mathrm{P}$ & & 0,005 & 0,5 & 0,7 & 1 & 15 & & & \\
\hline & & $\mathrm{C}$ & 1,37321 & 0,30316 & $-8,29157$ & 12,7970 & $-6,10070$ & 0,84758 & & 96,9 & 0,0544 \\
\hline & $25+30$ & $\mathrm{P}$ & & 0,4 & 10 & & & & & & \\
\hline & & $\mathrm{C}$ & 1,37981 & $-1,01369$ & $-0,80823$ & & & & & 96,8 & 0,057 \\
\hline & $30+35$ & $\mathrm{P}$ & & 0,4 & 0,5 & 5 & & & & & \\
\hline & & $\mathrm{C}$ & 1,41644 & $-2,00139$ & 1,03858 & $-0,48754$ & & & & 95,1 & 0,0703 \\
\hline & $35+40$ & $\mathrm{P}$ & & 0,4 & 2 & 4 & 15 & 25 & & & \\
\hline & & $\mathrm{C}$ & 1,30905 & $-0,90914$ & 0,61660 & $-1,71640$ & 3,29209 & $-3,00669$ & & 97,1 & 0,0532 \\
\hline & $40+45$ & $\mathrm{P}$ & & 0,5 & 0,6 & 5 & 10 & & & & \\
\hline & & $\mathrm{C}$ & 1,32531 & $-3,09304$ & 2,38364 & $-1,58599$ & 1,64211 & & & 97,3 & 0,0509 \\
\hline
\end{tabular}

Em que: $\mathrm{R}=$ Região; $\mathrm{CLD}=$ Classes de diâmetro; $\mathrm{P}=$ Potência; $\mathrm{C}=$ Coeficiente.

\section{Polinômio de $5^{\circ}$ grau}

Mediante a análise das medidas de precisão, verificou-se, nas Tabelas 7 e 8, que todos os modelos apresentam ajustes satisfatórios. É importante observar que as estatísticas tradicionais ou medidas de precisão apresentadas nas Tabelas 7 e 8 não retratam o desempenho das funções de afilamento, considerando que são médias que não consideram a posição da estimativa ao longo dos fustes. Portanto, servem apenas como um indicativo da correlação existentes entre as variáveis envolvidas nos modelos testados. Nesse contexto, entre os polinômios, o menor coeficiente de determinação nas regiões foi de $93,5 \%$ e o maior de $98,1 \%$; para os sítios o menor foi de $94,6 \%$ e o maior 99,0\%. Verificou-se por meio desses dados, juntamente com o erro padrão residual, uma ligeira superioridade no ajuste por sítio, em relação aos ajustes por região.

TABELA 7: Parâmetros estimados e medidas de precisão do polinômio do $5^{\circ}$ grau por classe de diâmetro e por sítio.

\begin{tabular}{c|c|c|c|c|c|c|c|c|c}
\hline S & CLD & \multicolumn{8}{c}{ Parâmetros estimados } \\
\cline { 3 - 11 } & $(\mathrm{cm})$ & $\beta_{0}$ & $\beta_{1}$ & $\beta_{2}$ & $\beta_{3}$ & $\beta_{4}$ & $\beta_{5}$ & $\mathrm{R}^{2}$ & Syx \\
\hline \multirow{4}{*}{ II } & $15+20$ & 1,31770 & $-5,39165$ & 26,44546 & $-67,35236$ & 76,43094 & $-31,56774$ & 96,9 & 0,0511 \\
& $20-25$ & 1,30698 & $-5,56646$ & 27,17428 & $-66,03874$ & 70,64478 & $-27,52905$ & 96,8 & 0,0549 \\
& $25-30$ & 1,24343 & $-4,94079$ & 23,41133 & $-56,33864$ & 59,80093 & $-23,20535$ & 96,3 & 0,0588 \\
& $30+35$ & 1,24095 & $-5,66650$ & 30,20954 & $-79,06375$ & 92,18947 & $-39,56691$ & 95,4 & 0,0654 \\
\hline
\end{tabular}

Continua ... 
TABELA 7: Continuação ...

\begin{tabular}{|c|c|c|c|c|c|c|c|c|c|}
\hline \multirow[t]{2}{*}{$\mathrm{S}$} & \multirow{2}{*}{$\begin{array}{l}\text { CLD } \\
(\mathrm{cm})\end{array}$} & \multicolumn{8}{|c|}{ Parâmetros estimados } \\
\hline & & $\beta_{0}$ & $\beta_{1}$ & $\beta_{2}$ & $\beta_{3}$ & $\beta_{4}$ & $\beta_{5}$ & $\mathrm{R}^{2}$ & Syx \\
\hline \multirow[t]{3}{*}{ II } & \begin{tabular}{lll}
35 & 40 \\
40 & \multicolumn{1}{|c}{45} \\
\end{tabular} & $\begin{array}{l}1,20225 \\
1,27905\end{array}$ & $\begin{array}{l}-4,59156 \\
-6,76849\end{array}$ & $\begin{array}{l}20,47675 \\
36,49065\end{array}$ & $\begin{array}{r}-44,18974 \\
-89,84488\end{array}$ & $\begin{array}{l}41,33877 \\
97,71952\end{array}$ & $\begin{array}{l}-14,10766 \\
-39,39822\end{array}$ & $\begin{array}{l}97,3 \\
97,7\end{array}$ & $\begin{array}{l}0,0484 \\
0,0479\end{array}$ \\
\hline & 15 卜 20 & 1,29092 & $-5,18638$ & 26,19238 & $-66,33118$ & 73,81466 & $-29,81934$ & 95,5 & 0,0612 \\
\hline & $20 \div 25$ & 1,25443 & $-4,76507$ & 22,65010 & $-55,52240$ & 59,93213 & $-23,506300$ & 95,4 & 0,0630 \\
\hline \multirow[t]{6}{*}{ III } & -30 & 1,22552 & $-4,93013$ & 25,19409 & $-65,18744$ & 75,22046 & $-31,96124$ & 97,2 & 0,0495 \\
\hline & $30 \div 35$ & 1,24793 & $-5,64903$ & 30,53238 & $-79,07905$ & 90,81680 & $-38,50182$ & 96,6 & 0,0547 \\
\hline & $35 \div 40$ & 1,21108 & $-4,02500$ & 13,38945 & $-17,48409$ & 2,55458 & 5,00745 & 95,5 & 0,0637 \\
\hline & $40 \vdash 45$ & 1,20346 & $-4,55066$ & 21,61636 & $-48,57913$ & 46,69421 & $-16,32903$ & 97,0 & 0,0524 \\
\hline & 15 卜 20 & 1,34445 & $-6,63240$ & 34,37428 & $-85,66833$ & 94,82491 & $-38,45331$ & 96,4 & 0,0597 \\
\hline & $20 \vdash 25$ & 1,27441 & $-5,69238$ & 30,54216 & $-79,12898$ & 90,24163 & $-37,60163$ & 96,9 & 0,0513 \\
\hline \multirow[t]{6}{*}{ IV } & $25+30$ & 1,26999 & $-5,80113$ & 31,32526 & $-80,40033$ & 91,04286 & $-37,93907$ & 97,5 & 0,0473 \\
\hline & $30 \div 35$ & 1,25772 & $-5,7358$ & 32,16274 & $-85,07113$ & 99,03664 & $-42,39418$ & 96,3 & 0,0576 \\
\hline & $35 \vdash 40$ & 1,21837 & $-4,70008$ & 24,56249 & $-61,69163$ & 68,26714 & $-28,11570$ & 97,7 & 0,0433 \\
\hline & $40 \vdash 45$ & 1,28425 & $-5,94458$ & 30,29979 & $-73,02179$ & 77,78199 & $-30,76483$ & 94,6 & 0,0764 \\
\hline & 15 ト 20 & 1,35554 & $-6,49678$ & 38,13987 & $-111,08391$ & 144,6057 & $-69,06667$ & 99,0 & 0,0326 \\
\hline & $20 \div 25$ & 1,27084 & $-5,27429$ & 27,22446 & $-69,23847$ & 77,89500 & $-32,20617$ & 96,1 & 0,0575 \\
\hline \multirow[t]{4}{*}{ V } & $25 \div 30$ & 1,26300 & $-5,23965$ & 26,47442 & $-66,18782$ & 73,98809 & $-30,73612$ & 97,5 & 0,0475 \\
\hline & $30 \vdash 35$ & 1,24431 & $-4,76593$ & 21,97108 & $-49,16444$ & 47,68862 & $-16,87994$ & 97,0 & 0,0533 \\
\hline & $35 \vdash 40$ & 1,24446 & $-4,93878$ & 24,48276 & $-61,34544$ & 69,63937 & $-29,69490$ & 97,9 & 0,0444 \\
\hline & $40 \vdash 45$ & 1,19472 & $-3,84751$ & 17,01557 & $-36,36726$ & 33,74122 & $-11,88964$ & 98,7 & 0,0362 \\
\hline
\end{tabular}

Em que: $\mathrm{S}=$ Classes de sítio; $\mathrm{CLD}=$ Classes de diâmetro.

TABELA 8: Parâmetros estimados e medidas de precisão do polinômio do $5^{\circ}$ grau por classe de diâmetro e por região.

\begin{tabular}{|c|c|c|c|c|c|c|c|c|c|c|}
\hline \multirow[t]{2}{*}{$\mathrm{R}$} & \multirow{2}{*}{\multicolumn{2}{|c|}{$\begin{array}{l}\text { CLD } \\
(\mathrm{cm})\end{array}$}} & \multicolumn{8}{|c|}{ Parâmetros estimados } \\
\hline & & & $\beta_{0}$ & $\beta_{1}$ & $\beta_{2}$ & $\beta_{3}$ & $\beta_{4}$ & $\beta_{5}$ & $\mathrm{R}^{2}$ & Syx \\
\hline \multirow{6}{*}{1} & 15 & -20 & 1,30982 & $-6,12951$ & 30,01702 & $-73,00982$ & 80,23661 & $-32,57719$ & 98,1 & 0,043 \\
\hline & 20 & 25 & 1,24909 & $-5,07885$ & 24,41553 & $-60,04343$ & 66,54944 & $-27,27288$ & 96,5 & 0,054 \\
\hline & 25 & -30 & 1,22431 & $-4,98548$ & 25,88248 & $-67,01064$ & 77,69547 & $-33,30287$ & 97,3 & 0,046 \\
\hline & 30 & 35 & 1,20518 & $-4,57938$ & 24,41228 & $-64,05771$ & 74,54750 & $-32,09112$ & 97,5 & 0,044 \\
\hline & 35 & -40 & 1,22857 & $-5,18264$ & 28,56081 & $-76,19731$ & 90,47826 & $-39,75219$ & 97,2 & 0,047 \\
\hline & 40 & -45 & 1,22102 & $-5,22384$ & 26,70358 & $-63,75783$ & 66,59111 & $-25,78177$ & 98,0 & 0,041 \\
\hline \multirow{6}{*}{2} & 15 & 20 & 1,28467 & $-5,02425$ & 24,42467 & $-63,18803$ & 73,28536 & $-31,01101$ & 96,5 & 0,056 \\
\hline & 20 & 25 & 1,24029 & $-4,83142$ & 24,54140 & $-63,65991$ & 72,05670 & $-29,51001$ & 97,4 & 0,046 \\
\hline & 25 & 30 & 1,22941 & $-5,02303$ & 25,11766 & $-63,45898$ & 70,94355 & $-29,01677$ & 97,1 & 0,050 \\
\hline & 30 & 35 & 1,21722 & $-4,81908$ & 21,50333 & $-47,17869$ & 45,83246 & $-16,48380$ & 96,0 & 0,060 \\
\hline & 35 & 40 & 1,20088 & $-4,44366$ & 19,72046 & $-43,56375$ & 42,19196 & $-15,04988$ & 97,3 & 0,049 \\
\hline & 40 & -45 & 1,21330 & $-4,14548$ & 18,76878 & $-41,80241$ & 39,91351 & $-13,84345$ & 93,5 & 0,081 \\
\hline \multirow{6}{*}{3} & 15 & -20 & 1,30660 & $-5,45294$ & 29,13069 & $-78,41966$ & 93,24454 & $-40,55934$ & 96,4 & 0,056 \\
\hline & 20 & 25 & 1,28307 & $-5,03621$ & 25,38813 & $-63,53378$ & 68,81671 & $-26,90792$ & 96,2 & 0,060 \\
\hline & 25 & -30 & 1,24302 & $-4,62072$ & 22,01238 & $-52,83758$ & 56,06442 & $-22,04328$ & 97,0 & 0,052 \\
\hline & 30 & 35 & 1,25751 & $-5,23931$ & 27,93353 & $-71,24270$ & 79,84484 & $-33,03035$ & 97,6 & 0,047 \\
\hline & 35 & 40 & 1,20147 & $-3,56487$ & 10,85930 & $-10,36674$ & $-7,56086$ & 10,38066 & 97,2 & 0,049 \\
\hline & 40 & -45 & 1,23397 & $-5,54588$ & 30,70704 & $-79,08239$ & 89,04368 & $-36,99037$ & 97,5 & 0,049 \\
\hline 4 & 15 & 20 & 1,43327 & $-9,05279$ & 58,62532 & $-176,78797$ & 236,7333 & $-116,14314$ & 97,9 & 0,051 \\
\hline
\end{tabular}


TABELA 8: Continuação ...

\begin{tabular}{|c|c|c|c|c|c|c|c|c|c|c|}
\hline \multirow[t]{2}{*}{$\mathrm{R}$} & \multirow{2}{*}{\multicolumn{2}{|c|}{$\begin{array}{l}\text { CLD } \\
(\mathrm{cm})\end{array}$}} & \multicolumn{8}{|c|}{ Parâmetros estimados } \\
\hline & & & $\beta_{0}$ & $\beta_{1}$ & $\beta_{2}$ & $\beta_{3}$ & $\beta_{4}$ & $\beta_{5}$ & $\mathrm{R}^{2}$ & Syx \\
\hline \multirow{5}{*}{4} & 20 & -25 & 1,27275 & $-5,64085$ & 29,89296 & $-77,33829$ & 88,57081 & $-37,25055$ & 97,0 & 0,050 \\
\hline & 25 & 30 & 1,27323 & $-5,70508$ & 29,70447 & $-75,28823$ & 84,62023 & $-35,07348$ & 97,6 & 0,048 \\
\hline & 30 & 35 & 1,26889 & $-6,03493$ & 32,99073 & $-85,49482$ & 97,30211 & $-40,63670$ & 97,5 & 0,050 \\
\hline & 35 & 40 & 1,22329 & $-4,86555$ & 22,37025 & $-49,16218$ & 47,19812 & $-16,75006$ & 97,8 & 0,044 \\
\hline & 40 & 45 & 1,24004 & $-5,66507$ & 29,63965 & $-72,84870$ & 78,95705 & $-31,81597$ & 97,4 & 0,051 \\
\hline \multirow{6}{*}{5} & 15 & 20 & 1,31893 & $-5,53190$ & 27,92046 & $-71,14551$ & 80,60524 & $-33,34095$ & 96,6 & 0,053 \\
\hline & 20 & 25 & 1,32967 & $-5,74575$ & 28,02408 & $-67,54133$ & 72,07413 & $-28,19348$ & 96,5 & 0,058 \\
\hline & 25 & 30 & 1,31843 & $-6,54838$ & 34,93596 & $-90,39291$ & 104,4968 & $-44,52080$ & 96,6 & 0,060 \\
\hline & 30 & 35 & 1,32020 & $-7,32020$ & 40,31923 & $-104,67384$ & 120,5014 & $-50,90979$ & 93,9 & 0,079 \\
\hline & 35 & 40 & 1,23661 & $-4,66258$ & 19,61719 & $-38,48883$ & 31,05368 & $-8,48533$ & 96,7 & 0,057 \\
\hline & 40 & 45 & 1,25872 & $-5,96724$ & 31,70667 & $-78,70090$ & 86,13255 & $-34,87725$ & 97,0 & 0,054 \\
\hline
\end{tabular}

Em que: $\mathrm{R}=$ Região; CLD = Classes de diâmetro.

\section{Ajuste das razões entre volumes}

Com relação à equação de volume total, pode-se observar, na Tabela 9, que as medidas de precisão do modelo de Schumacher e Hall não diferem demasiadamente, quando o controle da base de dados foi por sítio ou por região.

TABELA 9: Parâmetros estimados e medidas de precisão do modelo de Clutter para equação de volume total, para cinco regiões e quatro sítios.

\begin{tabular}{|c|c|c|c|c|c|}
\hline \multirow[t]{2}{*}{ Local } & \multirow[t]{2}{*}{ Variável } & \multicolumn{4}{|c|}{ Parâmetros estimados } \\
\hline & & $\beta_{0}$ & $\beta_{1}$ & $\beta_{2}$ & $\mathrm{R}^{2}$ \\
\hline \multirow{5}{*}{ Região } & 1 & 0,00003457 & 1,87286322 & 1,16406418 & 92,7 \\
\hline & 2 & 0,00003080 & 2,20580733 & 0,80767302 & 98,8 \\
\hline & 3 & 0,00007232 & 1,77864158 & 1,03175808 & 99,0 \\
\hline & 4 & 0,00004801 & 2,12116087 & 0,75666920 & 98,0 \\
\hline & 5 & 0,00004237 & 1,82482019 & 1,14855720 & 99,0 \\
\hline \multirow{4}{*}{ Sítio } & II & 0,00003000 & 2,2200000 & 0,79630000 & 99,2 \\
\hline & III & 0,00003400 & 1,9400000 & 1,07630000 & 97,8 \\
\hline & IV & 0,00005223 & 1,9309273 & 0,96694538 & 97,7 \\
\hline & $\mathrm{V}$ & 0,00003018 & 2,0815069 & 0,96718861 & 98,1 \\
\hline
\end{tabular}

Com relação às razões entre volumes, pode-se observar, nas Tabelas 10, 11 e 12, que suas estatísticas são bastante satisfatórias. Como esperado, o coeficiente de determinação do modelo de CLUTTER (1980) e o de AMATEIS e BURKHART (1987) são ligeiramente superiores por sítio em relação aos ajustes por região, pelos mesmos motivos já apresentados para os modelos polinomiais.

A alta correlação obtida dá-se em razão dos modelos estabelecerem relação entre os volumes comerciais e o volume total. Em qualquer circunstância, a correlação entre o volume total e o parcial, para uma mesma árvore, sempre será muito forte, haja vista o controle implícito das fontes 
de variação que afetam tais variáveis.

TABELA 10: Parâmetros estimados e medidas de precisão do modelo de Clutter para a equação de volume comercial, por região e sítio.

\begin{tabular}{|c|c|c|c|c|c|}
\hline \multirow[t]{2}{*}{ Local } & \multirow[t]{2}{*}{ Variável } & \multicolumn{4}{|c|}{ Parâmetros estimados } \\
\hline & & $\beta_{0}$ & $\beta_{1}$ & $\beta_{2}$ & $\mathrm{R}^{2}$ \\
\hline \multirow{5}{*}{ Região } & 1 & 0,05224703 & 2,94257248 & $-2,21220236$ & 96,8 \\
\hline & 2 & 3,50539009 & 3,84897242 & $-4,19623205$ & 99,4 \\
\hline & 3 & 0,07200000 & 4,22000000 & $-3,48348000$ & 99,4 \\
\hline & 4 & 0,72706735 & 3,96187775 & $-3,86125391$ & 99,2 \\
\hline & 5 & 0,61814774 & 4,15286182 & $-3,98621905$ & 99,4 \\
\hline \multirow{4}{*}{ Sítio } & II & 4,72200000 & 3,83000000 & $-4,24348000$ & 99,6 \\
\hline & III & 0,47047407 & 3,97817867 & $-3,76120094$ & 98,9 \\
\hline & IV & 0,86265984 & 3,99354696 & $-3,96332183$ & 99,4 \\
\hline & V & 0,88129020 & 3,98555430 & $-3,96523964$ & 99,5 \\
\hline
\end{tabular}

TABELA 11: Parâmetros estimados e medidas de precisão do modelo de Amateis e Burkhart para a equação de volume comercial até qualquer diâmetro superior, por região e sítio.

\begin{tabular}{|c|c|c|c|c|c|}
\hline \multirow[t]{2}{*}{ Local } & \multirow[t]{2}{*}{ Variável } & \multicolumn{4}{|c|}{ Parâmetros estimados } \\
\hline & & $\alpha_{0}$ & $\alpha_{1}$ & $\alpha_{2}$ & $\mathrm{R}^{2}$ \\
\hline \multirow{5}{*}{ Região } & 1 & 0,04057758 & 2,94539917 & 2,14498236 & 96,8 \\
\hline & 2 & 3,26305296 & 3,83403479 & 4,16321877 & 99,4 \\
\hline & 3 & 0,06688087 & 4,12945097 & 3,37642295 & 99,4 \\
\hline & 4 & 0,65681530 & 3,92354572 & 3,79790868 & 99,2 \\
\hline & 5 & 0,61422400 & 4,15680634 & 3,98816380 & 99,4 \\
\hline \multirow{4}{*}{ Sítio } & II & 4,51196067 & 3,75775867 & 4,16553505 & 99,6 \\
\hline & III & 0,43156407 & 3,92957047 & 3,69252133 & 98,9 \\
\hline & IV & 0,85805739 & 3,99256958 & 3,96097529 & 99,4 \\
\hline & $\mathrm{V}$ & 0,97702462 & 3,94769997 & 3,95863483 & 99,5 \\
\hline
\end{tabular}

TABELA 12: Parâmetros estimados e medidas de precisão do modelo de Amateis e Burkhart para a equação de volume comercial até qualquer altura, por região e sítio.

\begin{tabular}{|c|c|c|c|c|c|}
\hline \multirow[t]{2}{*}{ Local } & \multirow[t]{2}{*}{ Variável } & \multicolumn{4}{|c|}{ Parâmetros estimados } \\
\hline & & $\alpha_{0}$ & $\alpha_{1}$ & $\alpha_{2}$ & $\mathrm{R}^{2}$ \\
\hline \multirow{5}{*}{ Região } & 1 & 1,33252431 & 2,36016740 & 2,45190411 & 99,9 \\
\hline & 2 & 0,93364184 & 2,43184478 & 2,40997143 & 99,9 \\
\hline & 3 & 0,88711387 & 2,50208405 & 2,46460713 & 99,9 \\
\hline & 4 & 0,97149097 & 2,47859407 & 2,47262518 & 99,8 \\
\hline & 5 & 0,29204921 & 2,44442510 & 2,05788739 & 99,9 \\
\hline \multirow{4}{*}{ Sítio } & II & 0,77560255 & 2,41577120 & 2,33909862 & 99,9 \\
\hline & III & 0,54708470 & 2,44919028 & 2,26366851 & 99,8 \\
\hline & IV & 1,23536801 & 2,51688338 & 2,57636456 & 99,8 \\
\hline & $\mathrm{V}$ & 0,46275954 & 2,40258073 & 2,14885607 & 99,8 \\
\hline
\end{tabular}




\section{Exatidão das equações de afilamento para representar o perfil do tronco para as classes de diâmetro, nas regiões e sítios}

Nas Tabelas 13 e 14, são apresentadas, para as quatro equações estudadas, as estatísticas por altura relativa para as árvores compreendidas na menor $(20 \mid-24,9 \mathrm{~cm})$ e na maior $(35 \mid-39,9$ $\mathrm{cm}$ ) classe diamétrica respectivamente, para a região 1. Esses resultados permitiram inferir sobre a superioridade dos modelos polinomiais em relação às razões entre volumes, conforme detectado pela análise de desvio padrão das diferenças (SD), assim como da percentagem dos resíduos (RP) ao longo de todas as alturas relativas. Pode-se notar ainda que, para todas as classes diamétricas desta região, o polinômio de potências fracionárias e inteiras foi superior aos demais modelos testados, seguido de perto pelo desempenho também eficiente do polinômio do $5^{\circ}$ grau.

Das estatísticas mostradas nas Tabelas 13 e 14 e também para as outras duas classes diamétricas foi obtido o Ranking, para a região 1, conforme mostrado na Tabela 15 e para o sítio II na Tabela 16. Exemplificando: para a região 1 e para a classe diamétrica de 35 a $40 \mathrm{~cm}$ (Tabela 14), o Modelo 2, dentre os quatro analisados, apresentou para a altura relativa de $0 \%$ a primeira afirmação para a estatística $\mathrm{D}$, a segunda para a estatística SD, a primeira para a estatística SSRR e a primeira para a estatística RP e recebeu então a nota 5, conforme se observa na Tabela 15 . Na segunda posição, com nota 7 , ficou o Modelo 1, na terceira posição, com nota 12, ficou o Modelo 4 e, em último lugar, ficou o Modelo 3 com nota 16. Dessa maneira, na altura relativa de 0\%, o Modelo 2 foi o que apresentou estimativa mais acurada do fuste, para a classe diamétrica de 35 a 40 $\mathrm{cm}$ na região 1 .

TABELA 13: Estatísticas para as estimativas dos perfis da árvore de Pinus taeda na classe de 20 a $25 \mathrm{~cm}$ de diâmetro, para região 1, para os modelos testados com seus respectivos parâmetros: $\mathrm{D}=$ desvio; $\mathrm{SD}=$ desvio padrão das diferenças; $\mathrm{SSRR}=$ soma do quadrado das diferenças; $\mathrm{RP}=$ porcentagem dos resíduos.

\begin{tabular}{|c|c|c|c|c|c|c|c|c|c|c|c|c|c|c|c|c|c|c|}
\hline \multirow[t]{2}{*}{ Mod. } & \multirow[t]{2}{*}{ Est. } & \multicolumn{16}{|c|}{ Alturas relativas (\%) } & \multirow[t]{2}{*}{ Média } \\
\hline & & 0 & 1 & 2 & 3 & 4 & 5 & 10 & 15 & 25 & 35 & 45 & 55 & 65 & 75 & 85 & 95 & \\
\hline \multirow{4}{*}{1} & $\mathrm{D}$ & 0,56 & $-0,28$ & $-0,40$ & $-0,93$ & $-0,66$ & $-0,49$ & 0,53 & 0,20 & 0,24 & $-0,27$ & $-0,09$ & 0,24 & 0,03 & $-1,16$ & $-0,23$ & 0,00 & $-0,18$ \\
\hline & SD & 0,79 & 0,74 & 0,25 & 0,22 & 0,28 & 0,12 & 0,07 & 0,14 & 0,09 & 0,21 & 0,66 & 1,30 & 0,90 & 0,65 & 0,42 & 0,00 & 0,46 \\
\hline & SSRR & 0,008 & 0,009 & 0,004 & 0,01 & 0,008 & 0,004 & 0,004 & 0,004 & 0,003 & 0,008 & 0,02 & 0,058 & 0,057 & 0,19 & 0,06 & 0,00 & 0,03 \\
\hline & $\mathrm{RP}$ & 1,68 & $-1,27$ & $-1,59$ & $-3,78$ & $-2,78$ & $-2,07$ & 2,33 & 0,95 & 1,25 & $-1,66$ & $-1,01$ & 0,64 & $-0,80$ & $-14,48$ & $-3,27$ & 0,00 & $-1,72$ \\
\hline \multirow{4}{*}{2} & $\mathrm{D}$ & $-0,49$ & $-0,43$ & 0,13 & $-0,31$ & $-0,15$ & $-0,15$ & 0,09 & $-0,42$ & 0,20 & 0,12 & 0,08 & $-0,029$ & $-0,21$ & $-0,81$ & $-0,39$ & 0,00 & $-0,18$ \\
\hline & SD & 0,76 & 0,74 & 0,25 & 0,22 & 0,28 & 0,12 & 0,07 & 0,14 & 0,09 & 0,21 & 0,66 & 1,30 & 0,90 & 0,64 & 0,42 & 0,00 & 0,45 \\
\hline & SSRR & 0,009 & 0,01 & 0,003 & 0,004 & 0,004 & 0,002 & 0,001 & 0,005 & 0,002 & 0,006 & 0,02 & 0,06 & 0,06 & 0,13 & 0,06 & 0,00 & 0,03 \\
\hline & $\mathrm{RP}$ & $-1,87$ & $-1,80$ & 0,42 & $-1,34$ & $-0,72$ & $-0,68$ & 0,37 & $-2,08$ & 1,03 & 0,66 & 0,10 & $-1,41$ & $-2,89$ & $-10,49$ & $-4,92$ & 0,00 & $-1,71$ \\
\hline \multirow{4}{*}{3} & D & $-0,31$ & $-1,98$ & $-2,80$ & $-3,93$ & $-4,18$ & $-4,44$ & $-4,57$ & $-4,87$ & $-3,21$ & $-1,93$ & $-0,57$ & 0,64 & 1,58 & 1,91 & 3,57 & 0,00 & $-1,67$ \\
\hline & SD & 1,58 & 1,21 & 0,48 & 0,40 & 0,37 & 0,16 & 0,24 & 0,15 & 0,07 & 0,12 & 0,60 & 1,33 & 0,81 & 0,52 & 0,36 & 0,00 & 0,56 \\
\hline & SSRR & 0,01 & 0,04 & 0,06 & 0,13 & 0,16 & 0,18 & 0,22 & 0,29 & 0,14 & 0,07 & 0,03 & 0,06 & 0,12 & 0,25 & 0,87 & 0,00 & 0,17 \\
\hline & $\mathrm{RP}$ & $-1,40$ & $-7,48$ & $-10,68$ & $-15,83$ & $-17,21$ & $-18,64$ & $-20,72$ & $-23,98$ & $-17,04$ & $-11,43$ & $-4,14$ & 3,60 & 12,92 & 20,9 & 37,27 & 0,00 & $-4,92$ \\
\hline \multirow{4}{*}{4} & D & 0,20 & $-1,53$ & $-2,44$ & $-3,66$ & $-3,98$ & $-4,32$ & $-4,84$ & $-5,51$ & $-4,51$ & $-3,81$ & $-2,92$ & $-2,04$ & $-1,28$ & $-0,89$ & 1,15 & 0,00 & $-2,69$ \\
\hline & SD & 1,05 & 0,90 & 0,29 & 0,25 & 0,30 & 0,12 & 0,09 & 0,13 & 0,08 & 0,19 & 0,64 & 1,30 & 0,87 & 0,60 & 0,39 & 0,00 & 0,48 \\
\hline & SSRR & 0,01 & 0,03 & 0,05 & 0,11 & 0,14 & 0,17 & 0,24 & 0,37 & 0,29 & 0,26 & 0,22 & 0,22 & 0,14 & 0,14 & 0,11 & 0,00 & 0,17 \\
\hline & $\mathrm{RP}$ & 0,41 & $-5,85$ & $-9,31$ & $-14,70$ & $-16,38$ & $-18,13$ & $-21,88$ & $-27,06$ & $-23,91$ & $-22,47$ & $-19,52$ & $-16,53$ & $-12,40$ & $-11,48$ & 11,45 & 0,00 & $-13,84$ \\
\hline
\end{tabular}

Em que: 1 = Polinômio de quinto grau; 2 = Polinômio de potências fracionárias e inteiras; $3=$ Modelo de Clutter : $4=$ Modelo de Amateis e Burkhart. 
TABELA 14. Estatísticas para as estimativas dos perfis da árvore de Pinus taeda na classe de 35 a $40 \mathrm{~cm}$ de diâmetro, para região 1, para os modelos testados com seus respectivos parâmetros: $\mathrm{D}=$ desvio; $\mathrm{SD}=$ desvio padrão das diferenças; $\mathrm{SSRR}=$ soma do quadrado das diferenças; $\mathrm{RP}=$ porcentagem dos resíduos.

\begin{tabular}{|c|c|c|c|c|c|c|c|c|c|c|c|c|c|c|c|c|c|c|}
\hline \multirow[t]{2}{*}{ Mod. } & \multirow[t]{2}{*}{ Est. } & \multicolumn{16}{|c|}{ Alturas relativas (\%) } & \multirow[t]{2}{*}{ Média } \\
\hline & & 0 & 1 & 2 & 3 & 4 & 5 & 10 & 15 & 25 & 35 & 45 & 55 & 65 & 75 & 85 & 95 & \\
\hline & D & 3,27 & 1,70 & 0,11 & $-0,35$ & $-0,05$ & $-0,08$ & 1,48 & 1,09 & 0,51 & $-0,27$ & $-0,18$ & 0,26 & 0,03 & $-1,34$ & $-0,56$ & 0,00 & 0,38 \\
\hline \multirow[t]{4}{*}{1} & SD & 3,32 & 2,82 & 0,70 & 0,50 & 0,25 & 0,53 & 0,42 & 0,20 & 0,88 & 1,44 & 1,31 & 2,04 & 3,57 & 2,44 & 0,22 & 0,00 & 1,38 \\
\hline & SSRR & 0,03 & 0,02 & 0,003 & 0,003 & 0,001 & 0,003 & 0,01 & 0,007 & 0,008 & 0,016 & 0,02 & 0,03 & 0,10 & 0,17 & 0,05 & 0,00 & 0,03 \\
\hline & $\mathrm{RP}$ & 6,60 & 3,60 & 0,24 & $-0,92$ & $-0,10$ & $-0,22$ & 4,11 & 3,24 & 1,40 & $-1,30$ & $-1,13$ & 0,47 & $-1,68$ & $-10,65$ & $-6,92$ & 0,00 & $-0,22$ \\
\hline & $\mathrm{D}$ & 1,65 & 2,39 & 0,93 & 0,24 & 0,24 & $-0,12$ & 0,49 & 0,27 & 1,16 & 0,91 & 0,07 & $-0,59$ & $-0,68$ & $-0,69$ & $-0,64$ & 0,00 & 0,38 \\
\hline \multirow[t]{4}{*}{2} & SD & 3,36 & 2,81 & 0,68 & 0,49 & 0,24 & 0,53 & 0,41 & 0,19 & 0,90 & 1,47 & 1,32 & 2,03 & 3,56 & 2,43 & 0,22 & 0,00 & 1,38 \\
\hline & SSRR & 0,02 & 0,02 & 0,005 & 0,003 & 0,001 & $-0,003$ & 0,004 & 0,001 & 0,012 & 0,02 & 0,01 & 0,04 & 0,12 & 0,12 & 0,06 & 0,00 & 0,03 \\
\hline & $\mathrm{RP}$ & 3,24 & 5,13 & 2,22 & 0,59 & 0,63 & $-0,32$ & 1,32 & 0,77 & 3,51 & 2,81 & $-0,16$ & $-3,31$ & $-5,32$ & $-6,39$ & $-7,88$ & 0,00 & $-0,21$ \\
\hline & D & 13,14 & 10,1 & 7,32 & 5,80 & 5,22 & 4,44 & 4,07 & 3,74 & 5,45 & 6,61 & 7,30 & 7,88 & 8,25 & 7,28 & 4,11 & 0,00 & 6,72 \\
\hline \multirow[t]{4}{*}{3} & SD & 5,49 & 4,07 & 0,98 & 1,46 & 1,01 & 0,86 & 2,05 & 1,75 & 3,03 & 3,11 & 3,36 & 2,90 & 4,10 & 2,45 & 0,39 & 0,00 & 2,47 \\
\hline & SSRR & 0,38 & 0,26 & 0,16 & 0,11 & 0,09 & 0,07 & 0,07 & 0,07 & 0,16 & 0,27 & 0,41 & 0,59 & 0,86 & 1,11 & 1,08 & 0,00 & 0,38 \\
\hline & $\mathrm{RP}$ & 26,98 & 22,35 & 17,51 & 14,47 & 13,39 & 11,72 & 11,29 & 11,05 & 17,05 & 22,50 & 27,66 & 33,80 & 40,6 & 46,5 & 46,26 & 0,00 & 24,2 \\
\hline & $\mathrm{D}$ & 7,33 & 4,28 & 1,43 & $-0,14$ & $-0,75$ & $-1,58$ & $-2,14$ & $-2,63$ & $-1,16$ & $-0,12$ & 0,58 & 1,35 & 2,14 & 1,87 & $-0,14$ & 0,00 & 0,69 \\
\hline \multirow[t]{3}{*}{4} & SD & 3,39 & 2,77 & 0,37 & 0,41 & 0,09 & 0,27 & 0,56 & 0,34 & 1,26 & 1,77 & 1,83 & 2,21 & 3,71 & 2,38 & 0,26 & 0,00 & 1,44 \\
\hline & SSRR & 0,12 & 0,05 & 0,007 & 0,002 & 0,002 & 0,01 & 0,023 & 0,035 & 0,019 & 0,018 & 0,022 & 0,044 & 0,13 & 0,13 & 0,034 & 0,00 & 0,043 \\
\hline & $\mathrm{RP}$ & 14,99 & 9,33 & 3,39 & $-0,41$ & $-1,95$ & $-4,22$ & $-6,15$ & $-8,01$ & $-4,02$ & $-0,88$ & 1,72 & 5,17 & 9,14 & 10,64 & $-2,28$ & 0,00 & 1,76 \\
\hline
\end{tabular}

Em que: 1 = Polinômio de quinto grau; 2 = Polinômio de potências fracionárias e inteiras; 3 = Modelo de Clutter; $4=$ Modelo de Amateis e Burkhart.

TABELA 15: Ranking para as equações de afilamento por classe diamétrica, para a região 1.

\begin{tabular}{|c|c|c|c|c|c|c|c|c|c|c|c|c|c|c|c|c|}
\hline \multirow{3}{*}{$\begin{array}{r}\mathrm{H} \\
(\%) \\
\end{array}$} & \multicolumn{4}{|c|}{ Polinômio do $5^{\circ}$ Grau (1) } & \multicolumn{4}{|c|}{ PPFI (2) } & \multicolumn{4}{|c|}{ Clutter (3) } & \multicolumn{4}{|c|}{ Amateis e Burkhart (4) } \\
\hline & \multicolumn{16}{|c|}{ Classes Diamétricas $(\mathrm{cm})$} \\
\hline & $20-25$ & $25-30$ & $30-35$ & $35-40$ & $20-25$ & $25-30$ & $30-35$ & $35-40$ & $20-25$ & $25-30$ & $30-35$ & $35-40$ & $20-25$ & $25-30$ & $30-35$ & $35-40$ \\
\hline 0 & 10 & 7 & 8 & 7 & 10 & 14 & 4 & 5 & 11 & 12 & 16 & 16 & 8 & 7 & 12 & 12 \\
\hline 1 & 4 & 8 & 6 & 6 & 7 & 11 & 5 & 7 & 15 & 6 & 15 & 15 & 11 & 13 & 11 & 9 \\
\hline 2 & 7 & 10 & 7 & 6 & 4 & 6 & 5 & 8 & 15 & 10 & 16 & 16 & 11 & 14 & 12 & 10 \\
\hline 3 & 7 & 9 & 7 & 11 & 4 & 5 & 5 & 8 & 16 & 13 & 13 & 15 & 12 & 13 & 15 & 4 \\
\hline 4 & 7 & 10 & 7 & 6 & 4 & 6 & 5 & 7 & 15 & 11 & 13 & 15 & 11 & 13 & 15 & 9 \\
\hline 5 & 7 & 5 & 7 & 5 & 4 & 8 & 4 & 7 & 14 & 12 & 11 & 14 & 10 & 13 & 13 & 9 \\
\hline 10 & 7 & 7 & 7 & 8 & 4 & 4 & 5 & 4 & 12 & 11 & 13 & 16 & 14 & 13 & 15 & 12 \\
\hline 15 & 5 & 8 & 7 & 8 & 8 & 6 & 4 & 4 & 12 & 13 & 12 & 16 & 13 & 13 & 14 & 12 \\
\hline 25 & 9 & 5 & 4 & 4 & 6 & 7 & 6 & 8 & 10 & 9 & 14 & 15 & 14 & 12 & 10 & 11 \\
\hline 35 & 9 & 5 & 7 & 6 & 6 & 8 & 4 & 11 & 10 & 12 & 15 & 16 & 14 & 13 & 11 & 7 \\
\hline 45 & 8 & 6 & 8 & 7 & 7 & 9 & 4 & 5 & 10 & 14 & 14 & 16 & 14 & 10 & 9 & 12 \\
\hline 55 & 5 & 11 & 6 & 5 & 6 & 8 & 5 & 7 & 10 & 15 & 15 & 16 & 12 & 4 & 11 & 12 \\
\hline 65 & 6 & 10 & 5 & 5 & 9 & 6 & 6 & 7 & 12 & 15 & 15 & 16 & 12 & 7 & 11 & 12 \\
\hline 75 & 13 & 4 & 4 & 11 & 6 & 7 & 7 & 5 & 13 & 15 & 15 & 16 & 8 & 11 & 11 & 8 \\
\hline 85 & 6 & 4 & 6 & 7 & 8 & 7 & 10 & 10 & 12 & 15 & 15 & 15 & 10 & 11 & 6 & 5 \\
\hline
\end{tabular}

Em que: H = Altura; PPFI (2) = Polinômio de Potências Fracionárias e Inteiras (2).

Observando as estatísticas dos valores médios dos desvios (D), desvio padrão das diferenças (SD), soma do quadrado das diferenças (SSRR) e porcentagem dos resíduos (RP) para a equação de afilamento selecionada como a de melhor desempenho por região administrativa (Tabela 15) e por 
sítio (Tabela 16) pode-se observar menores valores dessas estatísticas, quando o controle da cubagem rigorosa foi por sítio. Esse mesmo comportamento foi observado, quando comparadas as estatísticas geradas pelos ajustes quando o controle da cubagem rigorosa foi por sítio em relação ao controle por região administrativa.

TABELA 16: Ranking para as equações de afilamento por classe diamétrica, para o sítio II.

\begin{tabular}{|c|c|c|c|c|c|c|c|c|c|c|c|c|c|c|c|c|}
\hline \multirow{3}{*}{$\begin{array}{r}\mathrm{H} \\
(\%) \\
\end{array}$} & \multicolumn{4}{|c|}{ Polinômio do $5^{\circ}$ Grau (1) } & \multicolumn{4}{|c|}{ PPFI (2) } & \multicolumn{4}{|c|}{ Clutter (3) } & \multicolumn{4}{|c|}{ Amateis e Burkhart (4) } \\
\hline & \multicolumn{16}{|c|}{ Classes Diamétricas $(\mathrm{cm})$} \\
\hline & $20-25$ & $25-30$ & $30-35$ & $35-40$ & $20-25$ & $25-30$ & $30-35$ & $35-40$ & $20-25$ & $25-30$ & $30-35$ & $35-40$ & $20-25$ & $25-30$ & $30-35$ & $35-40$ \\
\hline 0 & 7 & 7 & 9 & 8 & 7 & 5 & 4 & 4 & 14 & 13 & 11 & 13 & 12 & 14 & 16 & 15 \\
\hline 1 & 5 & 4 & 8 & 4 & 10 & 6 & 4 & 7 & 15 & 12 & 13 & 12 & 10 & 12 & 15 & 14 \\
\hline 2 & 6 & 4 & 8 & 7 & 8 & 7 & 4 & 5 & 14 & 12 & 13 & 13 & 9 & 13 & 15 & 15 \\
\hline 3 & 8 & 5 & 7 & 8 & 6 & 6 & 4 & 4 & 16 & 13 & 12 & 13 & 10 & 13 & 14 & 15 \\
\hline 4 & 8 & 7 & 7 & 8 & 4 & 4 & 4 & 4 & 15 & 13 & 12 & 13 & 13 & 13 & 14 & 15 \\
\hline 5 & 10 & 5 & 7 & 8 & 5 & 6 & 4 & 4 & 15 & 12 & 12 & 13 & 10 & 13 & 13 & 15 \\
\hline 10 & 10 & 7 & 9 & 11 & 5 & 5 & 5 & 6 & 15 & 13 & 10 & 10 & 10 & 14 & 13 & 7 \\
\hline 15 & 8 & 8 & 13 & 11 & 4 & 5 & 4 & 6 & 16 & 11 & 13 & 13 & 12 & 13 & 9 & 10 \\
\hline 25 & 4 & 8 & 7 & 6 & 8 & 6 & 4 & 9 & 15 & 13 & 13 & 13 & 12 & 5 & 9 & 11 \\
\hline 35 & 8 & 9 & 10 & 12 & 4 & 7 & 4 & 7 & 11 & 14 & 14 & 13 & 13 & 4 & 7 & 8 \\
\hline 45 & 9 & 9 & 9 & 11 & 6 & 5 & 6 & 5 & 8 & 13 & 12 & 12 & 13 & 6 & 10 & 9 \\
\hline 55 & 9 & 6 & 7 & 7 & 10 & 7 & 9 & 9 & 8 & 13 & 12 & 11 & 10 & 9 & 10 & 6 \\
\hline 65 & 5 & 8 & 7 & 5 & 8 & 7 & 12 & 9 & 11 & 12 & 13 & 13 & 13 & 10 & 8 & 12 \\
\hline 75 & 6 & 9 & 12 & 4 & 6 & 6 & 7 & 6 & 14 & 13 & 13 & 14 & 14 & 11 & 7 & 10 \\
\hline 85 & & 4 & 4 & 8 & & 11 & 7 & 4 & & 16 & 14 & 16 & & 9 & 10 & 12 \\
\hline
\end{tabular}

Em que: H = Altura; PPFI (2) = Polinômio de Potências Fracionárias e Inteiras (2).

Utilizando a Tabela 15 e os Rankings, gerados para as outras 4 regiões de estudo estruturou-se a Tabela 17. Da Tabela 16 e os demais Rankings gerados para as outras três classes de sítio estruturou-se a Tabela 18, respectivamente para as diferentes regiões e sítios onde são mostradas as equações que apresentaram as estatísticas mais acuradas dos diâmetros nas diferentes posições do fuste, de 0 a $85 \%$ da altura, por classe diamétrica.

A equação de potências fracionárias e inteiras apresentou estimativas mais acuradas em um maior número de casos para as cinco regiões e para as quatro classes de sítio estudadas. Exceção é feita para a primeira e última classe diamétrica nas regiões 2 e 4 respectivamente, e para a primeira classe diamétrica do sítio III. É importante ressaltar que nestas três condições a equação de potências fracionárias e inteiras foi a segunda mais acurada, comprovando a estabilidade dessa equação em estimar o diâmetro ao longo do fuste nas diferentes classes diamétricas, para as diferentes regiões e classes de sítio.

O polinômio do $5^{\circ}$ grau apresentou-se como a segunda equação com estimativas mais acuradas para as cinco regiões e para as quatro classes de sítio estudadas. Exceção é feita para a primeira e última classe diamétrica nas regiões 2 e 4 respectivamente, e para a primeira classe diamétrica do sítio III onde essa equação se apresentou com estimativas mais acuradas em um maior número de casos, superando o polinômio de potências fracionárias e inteiras.

Para a segunda e última classe diamétrica na região 2, para a terceira classe diamétrica na região 5 e para a terceira e segunda classe diamétrica nos sítios III e IV respectivamente, foi a 
equação de afilamento de Amateis e Burkhart que se apresentou como a segunda equação com estimativas mais acuradas. Para a maioria das outras situações a equação de afilamento de Amateis e Burkhart apresentou-se como a terceira colocada em relação à exatidão das estimativas, deixando o modelo de Clutter em quarto lugar.

TABELA 17: Equações com medidas mais acuradas dos diâmetros ao longo do fuste por classe diamétrica e região.

\begin{tabular}{|c|c|c|c|c|c|c|c|c|c|c|c|c|c|c|c|c|c|c|c|c|}
\hline \multirow{4}{*}{$\begin{array}{l}\mathrm{H} \\
(\%)\end{array}$} & \multicolumn{20}{|c|}{ Regiões } \\
\hline & \multicolumn{4}{|c|}{1} & \multicolumn{4}{|c|}{2} & \multicolumn{4}{|c|}{3} & \multicolumn{4}{|c|}{4} & \multicolumn{4}{|c|}{5} \\
\hline & \multicolumn{20}{|c|}{ Valor Central $(\mathrm{cm})$} \\
\hline & 22,5 & 27,5 & 32,5 & 37,5 & 22,5 & 27,5 & 32,5 & 37,5 & 22,5 & 27,5 & 32,5 & 37,5 & 22,5 & 27,5 & 32,5 & 37,5 & 22,5 & 27,5 & 32,5 & 37,5 \\
\hline 8 & 4 & $1-4$ & 2 & 2 & $1-2$ & 2 & 2 & 2 & 2 & 2 & 2 & 1 & 2 & 2 & 2 & 2 & $1-2$ & 2 & 2 & 2 \\
\hline 1 & 1 & 3 & 2 & 1 & 1 & 2 & 2 & 2 & 2 & 1 & 2 & 1 & 1 & 1 & 2 & 1 & 1 & 1 & 1 & $1-2$ \\
\hline 2 & 2 & 2 & 2 & 1 & 1 & 2 & 1 & 2 & 2 & 2 & 2 & 2 & 2 & 2 & 2 & 1 & 2 & 2 & 2 & 1 \\
\hline 3 & 2 & 2 & 2 & 4 & 1 & 2 & 2 & 2 & 2 & 2 & 2 & 2 & 2 & 2 & 2 & 1 & 2 & 2 & 2 & 2 \\
\hline 4 & 2 & 2 & 2 & 1 & 1 & 2 & 1 & 2 & 2 & 2 & 3 & 2 & 2 & 2 & 2 & 1 & 2 & 2 & 2 & 2 \\
\hline 5 & 2 & 1 & 2 & 1 & $1-4$ & 2 & 2 & 2 & 2 & 2 & 2 & 2 & 1 & $2-3$ & 2 & 2 & 2 & 1 & 3 & 2 \\
\hline 10 & 2 & 2 & 2 & 2 & 1 & $2-4$ & 2 & 2 & 1 & 4 & 2 & 4 & 2 & 2 & 2 & 2 & 2 & 3 & 3 & 2 \\
\hline 15 & 1 & 2 & 2 & 2 & 1 & 2 & 2 & 4 & 1 & 1 & 2 & 2 & 4 & 2 & 2 & 1 & 2 & 2 & $2-4$ & $2-4$ \\
\hline 25 & 2 & 1 & 1 & 1 & 3 & 2 & 1 & 4 & 2 & 2 & $1-2$ & 4 & 2 & $1-4$ & 1 & 1 & 2 & 4 & 4 & 1 \\
\hline 35 & 2 & 1 & 2 & 1 & 3 & $1-2$ & 1 & 1 & $2-4$ & 2 & 2 & 1 & 2 & 4 & 1 & 4 & 2 & 4 & 4 & 2 \\
\hline 45 & 2 & 1 & 2 & 2 & 2 & $1-3-4$ & 1 & 2 & 2 & 2 & 2 & 2 & 2 & 2 & 2 & 4 & 1 & $1-4$ & 2 & $1-2-4$ \\
\hline 55 & 1 & 4 & 2 & 1 & 2 & 3 & 2 & 2 & 2 & 1 & 1 & 1 & 1 & 1 & 1 & 4 & $2-4$ & 2 & $1-2-4$ & 4 \\
\hline 65 & 1 & 2 & 1 & 1 & 2 & 4 & 2 & 3 & 1 & 2 & 1 & 1 & 1 & 1 & 1 & 1 & 4 & 1 & $2-4$ & 2 \\
\hline 75 & 2 & 1 & 1 & 2 & 2 & 2 & 2 & 2 & 2 & 2 & 1 & 1 & 2 & 2 & 2 & 1 & 2 & 4 & 1 & 2 \\
\hline \multirow[t]{5}{*}{85} & 1 & 1 & $1-4$ & 4 & - & 2 & 1 & 2 & - & 1 & 2 & 2 & 1 & 1 & 1 & 2 & 1 & $1-2$ & 4 & 2 \\
\hline & $2(9)$ & 2(6) & $2(11)$ & $2(5)$ & $1(8)$ & $2(12)$ & $2(9)$ & $2(11)$ & 2(11) & $2(10)$ & $2(11)$ & $2(7)$ & $2(9)$ & $2(9)$ & $2(10)$ & $1(8)$ & $2(11)$ & $2(7)$ & $2(8)$ & $2(12)$ \\
\hline & $1(5)$ & $1(7)$ & $1(4)$ & $1(8)$ & $2(5)$ & $4(3)$ & $1(6)$ & $4(2)$ & 1(3) & $1(4)$ & $1(4)$ & $1(6)$ & $1(5)$ & $1(5)$ & $1(5)$ & 2(4) & $1(4)$ & $1(5)$ & $4(6)$ & $1(4)$ \\
\hline & $4(1)$ & $4(2)$ & $4(1)$ & $4(2)$ & $3(2)$ & $1(2)$ & & $3(1)$ & $4(1)$ & 4(1) & $3(1)$ & 4(2) & 4(1) & $4(2)$ & & 4(3) & $4(2)$ & 4(4) & $3(2)$ & $4(3)$ \\
\hline & & $3(1)$ & & & (4(1) & $3(2)$ & & $1(1)$ & & & & & & $3(1)$ & & & & $3(1)$ & 1(3) & \\
\hline
\end{tabular}

Em que: $($ ) = Número de casos de estimativa mais acuradas em primeiro lugar pelas equações 1, 2, 3 e 4 por classe de diâmetro; H = Altura

Nas Figuras 1, 2, 3 e 4, encontram-se representados os perfis médios das árvores em relação
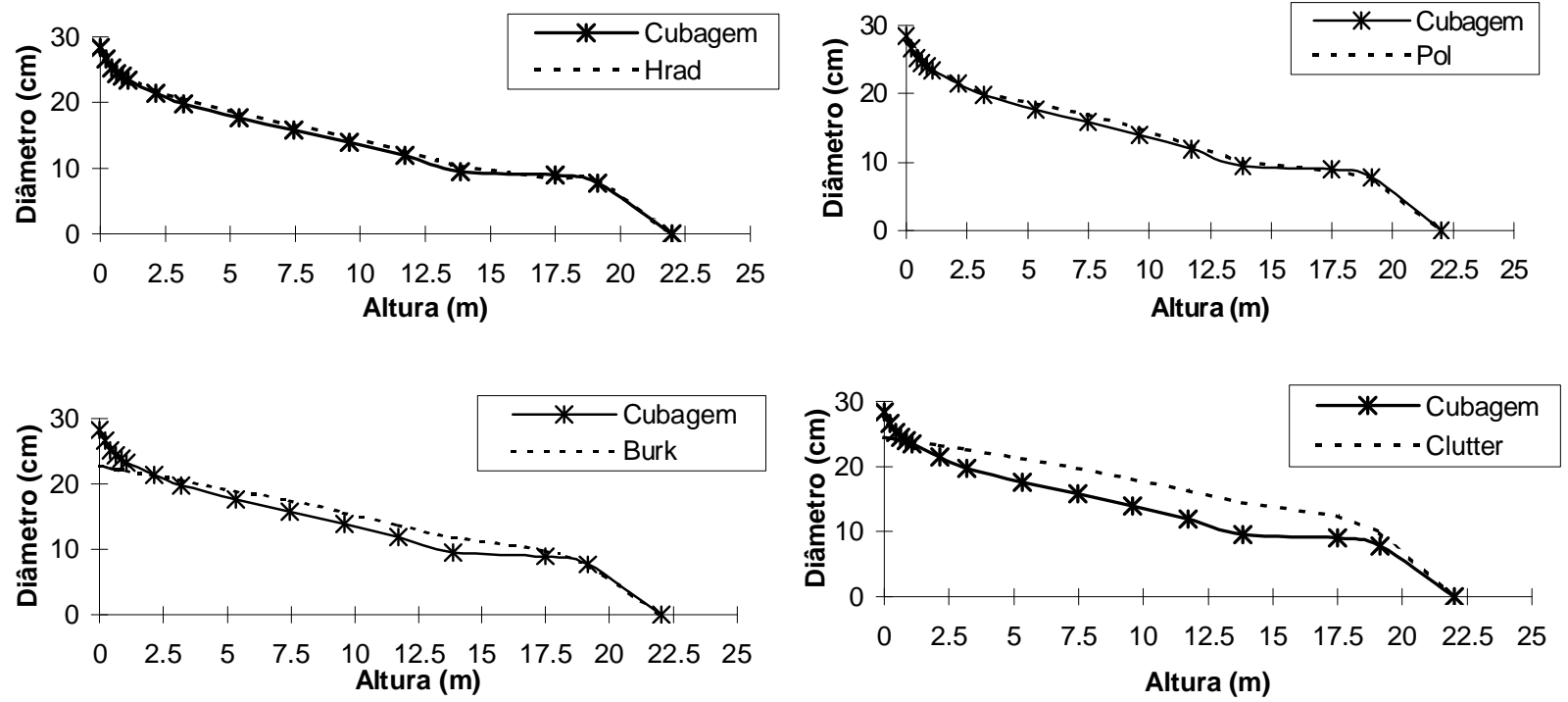

FIGURA 1: Perfil da árvore de Pinus taeda para a classe de diâmetro de 20 a 25 cm, para a classe de sítio II. 
TABELA 18: Equações com medidas mais acuradas dos diâmetros ao longo do fuste por classe diamétrica e sítio.

\begin{tabular}{|c|c|c|c|c|c|c|c|c|c|c|c|c|c|c|c|c|}
\hline \multirow[t]{3}{*}{$\mathrm{H}$} & \multicolumn{16}{|c|}{ Classes de Sítio } \\
\hline & \multicolumn{4}{|c|}{ II } & \multicolumn{4}{|c|}{ III } & \multicolumn{4}{|c|}{ IV } & \multicolumn{4}{|c|}{$\mathrm{V}$} \\
\hline & \multicolumn{16}{|c|}{ Valor Central da Classe $(\mathrm{cm})$} \\
\hline$(\%)$ & 22,5 & 27,5 & 32,5 & 37,5 & 22,5 & 27,5 & 32,5 & 37,5 & 22,5 & 27,5 & 32,5 & 37,5 & 22,5 & 27,5 & 32,5 & 37,5 \\
\hline 0 & $1-2$ & 2 & 2 & 2 & 1 & 2 & 2 & 2 & 2 & 2 & 2 & 2 & 2 & 2 & 2 & 2 \\
\hline 1 & 1 & 1 & 2 & 1 & 2 & 2 & 2 & 1 & 1 & 1 & 2 & 2 & 1 & 1 & 1 & 1 \\
\hline 2 & 1 & 1 & 2 & 2 & $2-3$ & 2 & 2 & 2 & 1 & 2 & 2 & 2 & 1 & 1 & 2 & 1 \\
\hline 3 & 2 & 1 & 2 & 2 & 3 & 2 & 3 & 2 & 2 & 2 & 2 & 2 & 1 & 1 & 3 & 2 \\
\hline 4 & 2 & 2 & 2 & 2 & 3 & 3 & 3 & 2 & 2 & 2 & 2 & 2 & 2 & 2 & 3 & 2 \\
\hline 5 & 2 & 1 & 2 & 2 & $2-3$ & 2 & 3 & 3 & 1 & 1 & 3 & 3 & 3 & 3 & 3 & 2 \\
\hline 10 & 2 & 2 & 2 & 2 & 1 & 4 & 2 & 1 & 4 & 3 & 2 & 2 & 2 & 2 & 2 & 1 \\
\hline 15 & 2 & 2 & 2 & 2 & 1 & 1 & 4 & 1 & 2 & 2 & 2 & 2 & 2 & 2 & 2 & 1 \\
\hline 25 & 1 & 4 & 2 & 1 & 2 & 2 & 4 & 2 & 1 & 1 & 4 & 1 & 1 & 1 & 1 & 2 \\
\hline 35 & 2 & 4 & 2 & 2 & 2 & 2 & 1 & 2 & 2 & 2 & 4 & 1 & 2 & 2 & 1 & 2 \\
\hline 45 & 2 & 2 & 2 & 2 & 2 & 1 & $1-4$ & 2 & 2 & 4 & 4 & 2 & 2 & 2 & 2 & $2-4$ \\
\hline 55 & 3 & 1 & 1 & 4 & 1 & 2 & 4 & 4 & 1 & $2-4$ & 1 & 2 & 2 & 2 & 1 & 1 \\
\hline 65 & 1 & 2 & 1 & 1 & 1 & 2 & 1 & 4 & 1 & 2 & $1-4$ & 2 & 1 & 1 & 1 & 4 \\
\hline 75 & $1-2$ & 2 & $2-4$ & 1 & 1 & 2 & $2-4$ & 2 & 2 & 2 & 2 & 2 & 2 & 2 & 2 & 2 \\
\hline \multirow[t]{5}{*}{85} & & 1 & 1 & 2 & 1 & $1-2$ & 1 & 2 & 4 & 1 & 1 & 1 & 1 & 1 & 2 & 1 \\
\hline & 2(9) & $2(7)$ & $2(12)$ & $2(10$ & 1(7) & $2(11$ & $2(5)$ & $2(9)$ & $2(7)$ & $2(9)$ & $2(8)$ & $2(11$ & $2(8)$ & $2(8)$ & $2(7)$ & $2(8)$ \\
\hline & $1(6)$ & $1(6)$ & 1(3) & 1(4) & $2(6)$ & $1(3)$ & $4(5)$ & 1(3) & $1(6)$ & $4(2)$ & $1(3)$ & $1(3)$ & $1(6)$ & $1(6)$ & $1(5)$ & $1(6)$ \\
\hline & $3(1)$ & $4(2)$ & $4(1)$ & $4(1)$ & 3(4) & $3(1)$ & 1(4) & $4(2)$ & $4(2)$ & $3(1)$ & $4(4)$ & $3(1)$ & 3(1) & $3(1)$ & $3(3)$ & $4(2)$ \\
\hline & & & & & & $4(1)$ & $3(3)$ & $3(1)$ & & & $3(1)$ & & & & & \\
\hline
\end{tabular}

Em que: ( ) = Número de casos de estimativa mais acuradas em primeiro lugar pelas equações $1,2,3$ e 4 por classe
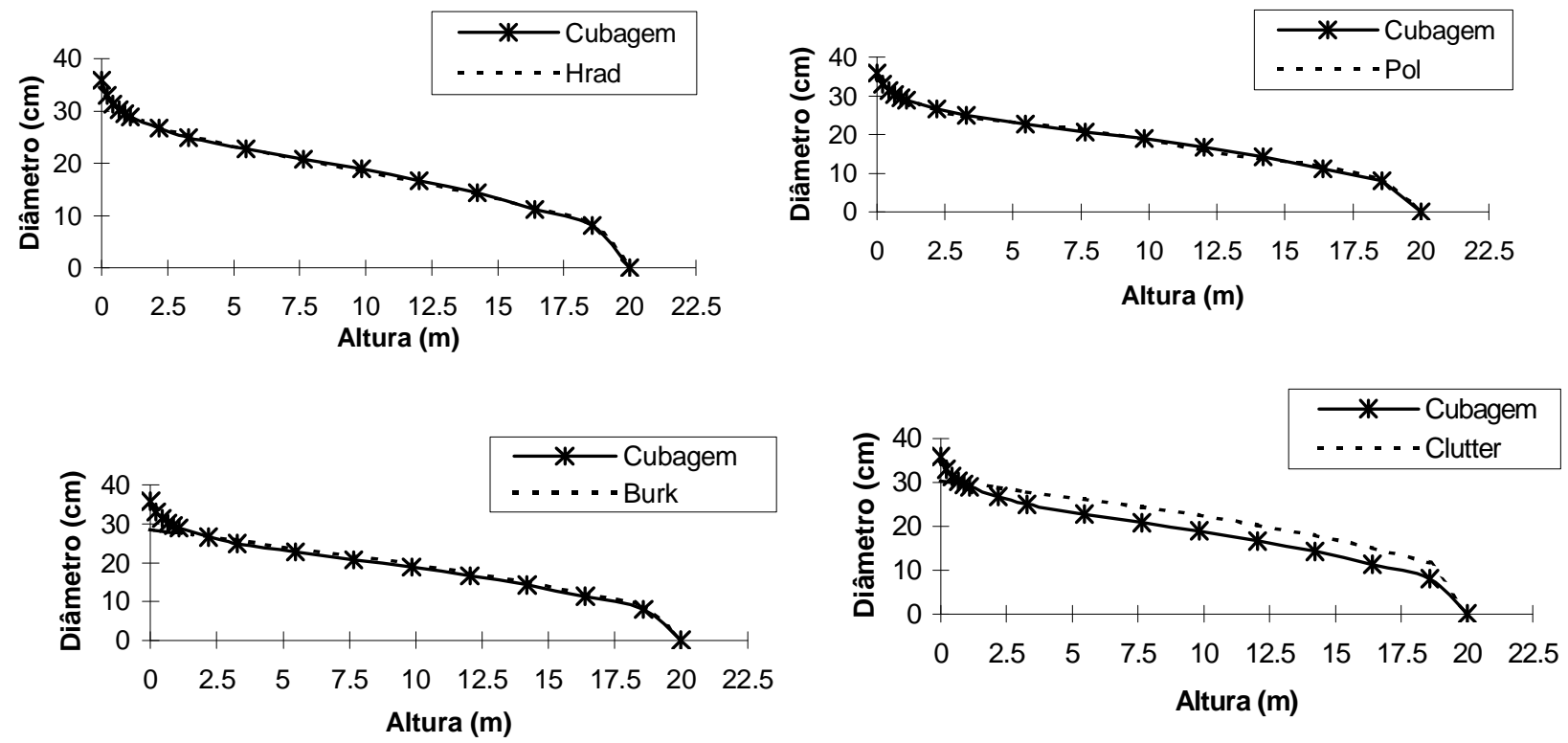

FIGURA 2: Perfil da árvore de Pinus taeda para a classe de diâmetro de 25 a $30 \mathrm{~cm}$, para a classe de sítio II. 

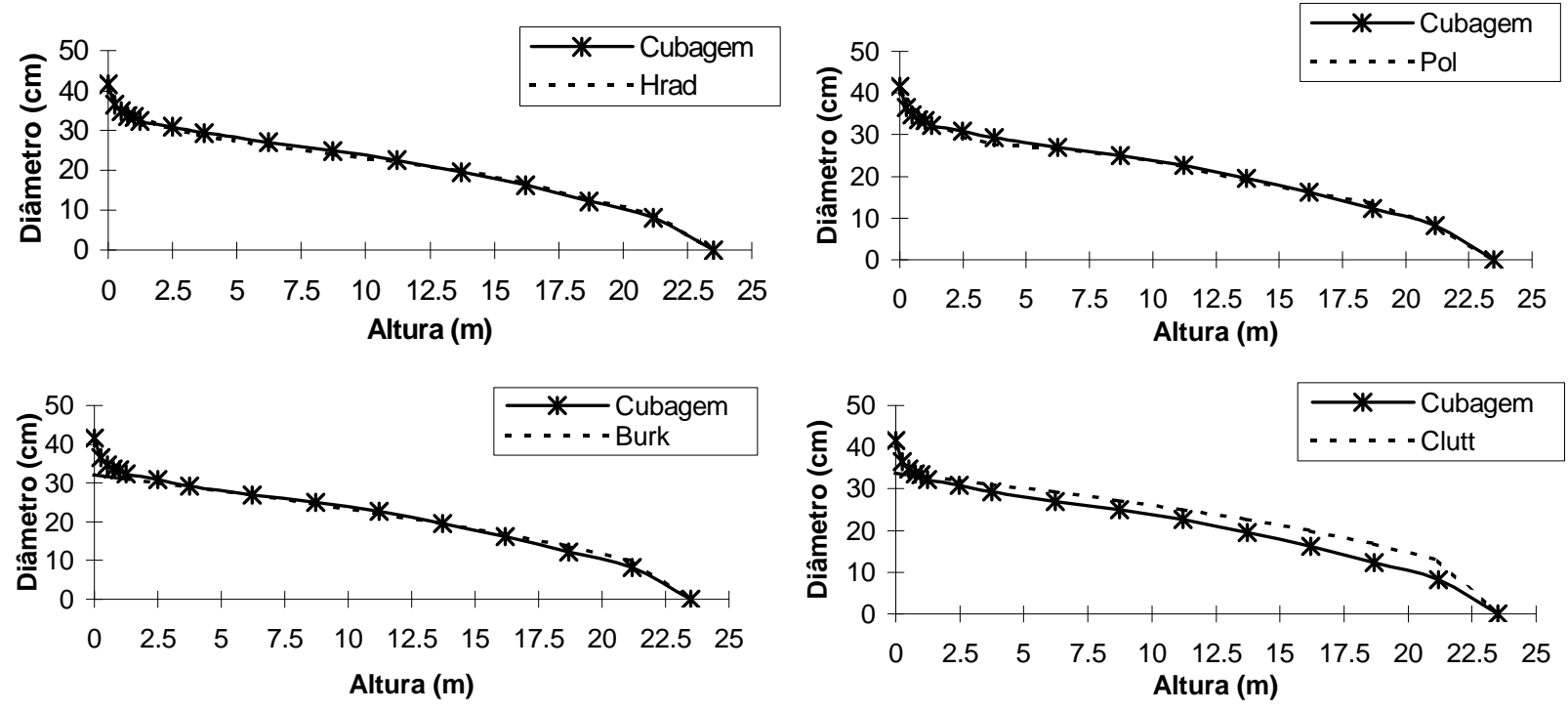

FIGURA 3: Perfil da árvore de Pinus taeda para a classe de diâmetro de 30 a 35 cm, para a classe de sítio II.
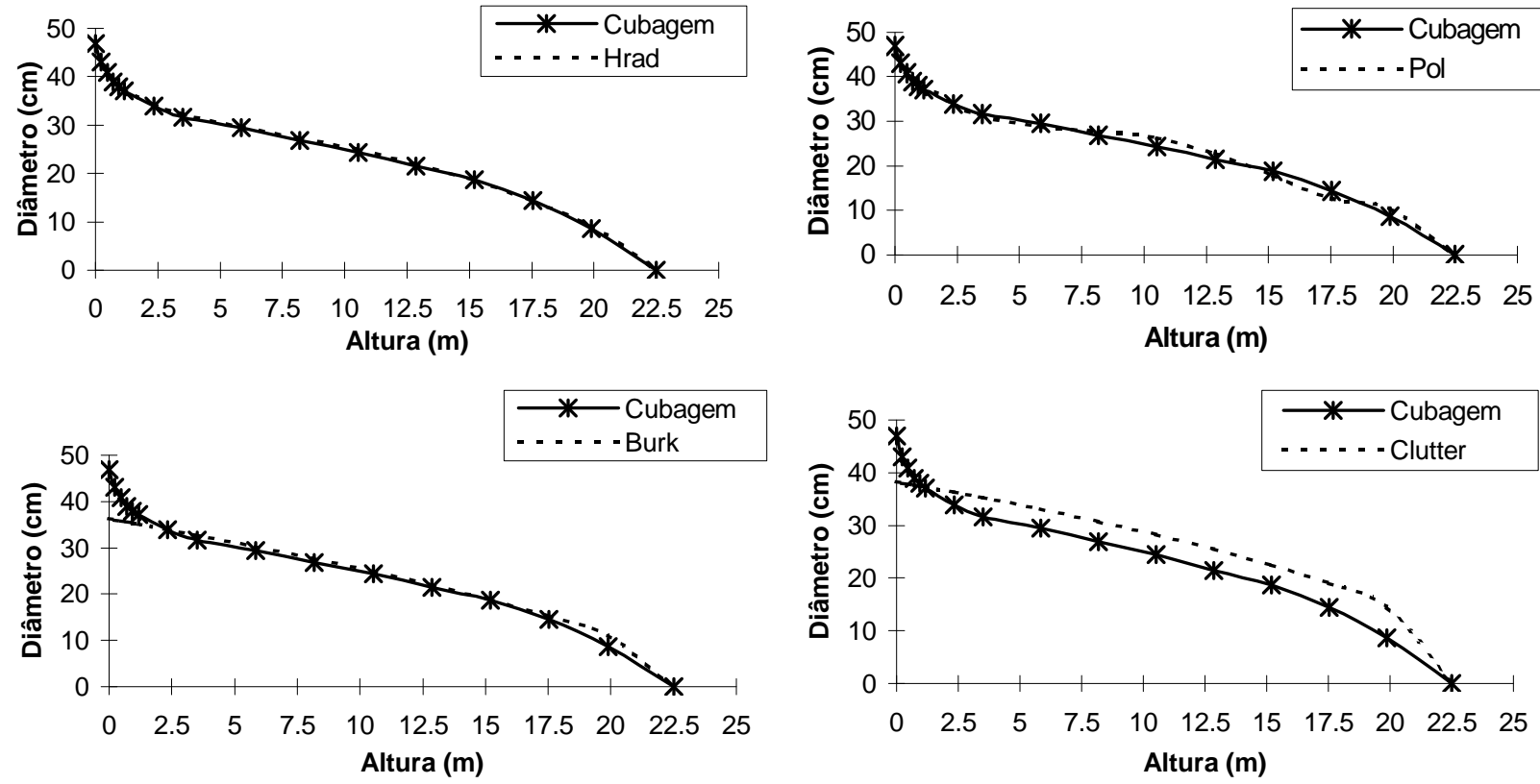

FIGURA 4: Perfil da árvore de Pinus taeda para a classe de diâmetro de 35 a 40 cm, para a classe de sítio II.

as estimativas dos diâmetros, pelos diferentes procedimentos para a classe de sítio II. Esses perfis corrobaoram os fatos discutidos anteriormente, permitindo visualizar a superioridade dos modelos polinomiais, destacando o modelo de potência fracionária e inteiras, em relação as frações de 
afilamento resultantes das razões entre volumes.

\section{CONCLUSÕES}

Verificou-se, em todos os casos, que os polinômios foram superiores às razões volumétricas para estimar o perfil dos troncos, com destaque especial para o modelo de potência fracionária.

Para as razões volumétricas, o modelo de melhor desempenho foi o de Amateis e Burkhart (1987), embora tenha apresentado tendências nas estimativas dos diâmetros da base (até 3 a $5 \%$ da altura) e do topo (acima de $75 \%$ da altura), na maioria dos casos estudados.

As regiões, sítios e classes diamétricas influenciaram na estimativa do perfil do tronco. fuste.

Os modelos ajustados por sítio tiveram melhores estimativas dos diâmetros ao longo do

Para o presente conjunto de dados, deve-se usar o modelo polinomial de potência fracionária, com ajuste por sítio e por classe diamétrica, para descrever o perfil do tronco.

\section{REFERÊNCIAS BIBLIOGRÁFICAS}

AMATEIS, R.L.; BURKHART, H.E. Cubic-foot volume equations for loblolly pine trees in cutover siteprepared plantations. Southern Journal of Applied Forestry, Bethesda, v. 11, n. 4, p.190-192, Nov.1987.

ANGELO, H.; CASTRO, L.H.R.; HOSOKAWA, R.T.; et al. Análise de componentes principais e função spline para definir a forma do tronco de Pinus tropicais. Floresta, Curitiba, v.25, n. 1-2, p. 55-67, jun./dez. 1995.

ASSIS, A.L.de. Acuracidade na estimativa de volumes comerciais de Eucalyptus grandis e Eucalyptus urophylla. Lavras: UFLA, 1998. 183p. Monografia.

CAMPOS, J.C.C.; LEITE, H.G.; OLIVEIRA, I.A. Variação da forma do tronco de clones de Eucalyptus, em duas regiões. In: CONGRESSO FLORESTAL BRASILEIRO, 7., CONGRESSO FLORESTAL PANAMERICANO, 1., 1993, Curitiba. Anais... Curitiba: SBS/SBEF, 1993. v.2, p. 559-565.

CAMPOS, J.C.C.; ZUNCONI, J.M.; RIBEIRO, J.C. Teste de um novo modelo para expressar "taper". In: SEMINÁRIO SOBRE ATUALIDADES E PERSPECTIVAS FLORESTAIS: o uso de funções de forma de tronco em estudos de volumetria de espécies florestais, 5., 1982, Curitiba. Anais... Curitiba: EMBRAPA/ URPFCS, 1982. p. 47-50.

CLUTTER, J.L. Development of taper functions from variable-top merchantable volume equations. Forest Science, Washington, v. 26, n. 1, p.117-120, Mar. 1980.

FERREIRA, S.O. Estudo da forma do fuste de Eucalyptus grandis e Eucalyptus cloeziana. 1999. 132p. (Dissertação - Mestrado em Engenharia Florestal) - Universidade Federal de Lavras, Lavras.

FIGUEIREDO FILHO, A.; BORDERS, B.E.; HITCH, K.L. Taper equations for Pinus taeda plantations in southern Brazil. Forest Ecology and Management, Amsterdam, v. 83, p.36-46, 1996. 
FIGUEIREDO FILHO, A.; OLIVEIRA, C.G. de; MOURA, J.B. de et al. Conversão de equações de volume em equações de forma compatíveis para Pinus elliottii. In: CONGRESSO FLORESTAL BRASILEIRO, 7., CONGRESSO FLORESTAL PANAMERICANO, 1., 1993, Curitiba. Anais... Curitiba: SBS/SBEF, v.2, p. 501-503, 1993.

FINGER, C.A.G.; ZANON, M.L.B.; SCHNEIDER, P.R.; et al. Funções de forma para Eucalyptus dunnii Maiden, implantados na depressão central e encosta sudeste do Rio Grande do Sul. Ciência Rural, Santa Maria, v.25, n.3, p. 399-403,1995.

FRIEDL, R.A. Dinâmica da forma dos fustes em povoamentos plantados de Araucária angustifolia (Bert) O. Ktze. 1989. 166p. Dissertação (Mestrado em Engenharia Florestal) - Universidade Federal de Santa Maria, Santa Maria.

FISCHER, F. Eficiência dos modelos polinomiais e das razões de volume na estimativa volumétrica dos sortimentos e do perfil do fuste de Pinus taeda. 1997. 167p. Dissertação (Mestrado em Engenharia Florestal) - Universidade Federal de Lavras, Lavras.

GARCIA, S.R.L.; LEITE, H.G.; YARED, J.A.G. Análise do perfil do tronco de Morototó (Didymopanax morototonï) em função do espaçamento. In: CONGRESSO FLORESTAL BRASILEIRO, 7., CONGRESSO FLORESTAL PANAMERICANO, 1., 1993, Curitiba. Anais... Curitiba: SBS/SBEF, 1993. v.2, p. 504-509.

GUIMARÃES. D.P.; LEITE, H.G. Um novo modelo para descrever o perfil do tronco. Revista Árvore, Viçosa, v. 16, n.2, p.170-180, maio/ago. 1992.

HOJER, A.G. Tallers och granenes tillraxt. Stocklan: Biran till Fr. Loven. Om vara barrskogar. 1903.

HRADETZKY, J. Analyse und interpretation statistisher abränger keiten. (Biometrische Beiträge zu aktuellen forschungs projekten). Baden: Württemberg Mitteilungen der FVA, Helf 76, 1976. 146p. (Abt. Biometric und Informatik, 21).

JORGE, L.A.B.; LARA, H.A. Programa de sortimento de madeira serrada de povoamentos de Pinus elliottii com alternativas de produtos padronizados. In: CONGRESSO FLORESTAL BRASILEIRO, 7., CONGRESSO FLORESTAL PANAMERICANO, 1., 1993, Curitiba. Anais... Curitiba: SBS/SBEF, 1993. v.2, p. 539-544.

LIMA, F.S. Análise de funções "taper" destinadas à avaliação de multiprodutos de árvores de Pinus elliottii. 1986. 70p. Dissertação (Mestrado em Ciências Florestais) - Universidade Federal de Viçosa, Viçosa.

PARRESOL, B.R.; HOTVEDT, J.E.; CAO, Q.V. Volume and taper prediction system for bald cypress. Canadian Journal of Forest Research, Otawa, v.17, p.250-259, 1987.

RIOS, M.S. A eficiência das funções polinomiais, da função spline cúbica e razões de volume para representar o perfil da árvore e estimar os sortimentos de Pinus elliottii. 1997. 116 p. Dissertação (Mestrado em Engenharia Florestal) - Universidade Federal de Lavras, Lavras.

ROSOT, M.A.D. Estudo comparativo de métodos para avaliação volumétrica por unidade de área em povoamento de Pinus taeda L. 1989. 163p. Dissertação (Mestrado em Engenharia Florestal) Universidade Federal de Curitiba, Curitiba.

SCHNEIDER, P.R. Forma de tronco e sortimentos para Pinus elliottii Engelm, da floresta nacional de Passo Fundo, Rio Grande do Sul. Acta Forest Brasil, Curitiba, n.1, p. 43-64, 1986. 
SCHOEPFER, W. Automatisierung des Massen, Sorten und Wertberechnung stenender Waldbestande Schriftenreihe Bad. [S.1.]: Wurtt-Forstl., 1966. n.p.

SCOLFORO, J.R.S. Modelo de prognose com simulador de desbaste para Pinus taeda e Pinus elliottii. Lavras: UFLA/FAEPE, 1997. 75p.

SCOLFORO, J.R.S.; RIOS, M.S.; OLIVEIRA, A.D. et al. Acuracidade de equações de afilamento para representar o perfil do fuste de Pinus elliottii. Revista Cerne, Lavras, v.4, n.1, p. 100-122, 1998.

SOARES, J.B. Otimização do sortimento de produtos florestais a partir de funções de distribuição diamétrica e funções de forma. 1993. 105p. Dissertação (Mestrado em Engenharia Florestal) Universidade Federal de Viçosa, Viçosa. 